\title{
Datenbasierte Weiterentwicklung des Kundenerlebnisses in der Möbelbranche
}

\author{
David Aemmer, Jonas Bigler und Deane Harder
}

\section{Zusammenfassung}

Das Konsumentenverhalten beim Kauf von Möbeln ist komplex. Konsequente Kundenorientierung entlang des Kaufentscheidungsprozesses findet nur statt, wenn kaufrelevante Informationen kundenzentriert bereitgestellt werden. Die vorliegende Studie untersucht entsprechende Erfolgsfaktoren im Schweizer Möbelhandel. Im Kaufprozess lag der Fokus auf den Schritten Informationssuche und Bewertung von Alternativen. Mit qualitativen Interviews wurde untersucht, wie Kunden vorgehen, welche Kanäle sie dabei nutzen und welchen Herausforderungen sie dabei begegnen (Customer Journey). Der 1. Schritt ist oft, dass sich Kunden zuerst online zu einem Möbelstück informieren, danach jedoch aufgrund fehlender, kaufrelevanter Information den stationären Handel besuchen. Es gilt ein Fällen des Kaufentscheides online zu erleichtern, Kanalwechsel motivierender zu gestalten und den Anschluss an Onlinerecherchen nahtlos zu gewährleisten. Im Zuge der voranschreitenden Digitalisierung, vor allem in den Bereichen Virtual Reality und Maschinenlernen, wird die Bedeutung dieser Faktoren weiter zunehmen.

D. Aemmer $(\bowtie)$

Bern, Schweiz

J. Bigler

Bern, Schweiz

D. Harder

Wirtschaft, Berner Fachhochschule, Bern, Schweiz

E-Mail: deane.harder@bfh.ch 


\subsection{Einleitung}

Im Zuge der digitalen Transformation kommt es in Wirtschaft und Gesellschaft zu tief greifenden Veränderungen (ITU 2018; Statista o. J.). Die Möglichkeiten des Internets beeinflussen das Konsumentenverhalten fundamental, da jederzeit und überall auf Informationen zurückgegriffen und Güter oder Dienstleistungen gekauft werden können (Heinemann et al. 2016, S. 453; Statista o. J.). Während der Kaufentscheidungsprozess früher meist im stationären Handel stattfand, werden Prozessschritte heute zunehmend online durchlaufen (Heinemann et al. 2016, S. 157 ff., 291 ff.). Dieses veränderte Verhalten stellt die Möbelbranche vor große Herausforderungen, obwohl - oder gerade weil - der stationäre Handel in dieser Branche noch hohe Relevanz hat. Kunden nutzen beim Möbelkauf klassischerweise erst digitale und anschließend physische Kanäle (Heinemann et al. 2016, S. 291 ff.; PwC 2017, S. 14). Deshalb ist es für die Möbelbranche wichtig, kaufrelevante Informationen kanalübergreifend zur Verfügung zu stellen. Entsprechend lautet die zentrale Forschungsfrage der vorliegenden Studie:

\section{Was sind die Erfolgsfaktoren für die kundenzentrierte Bereitstellung kaufrelevanter Informationen im Schweizer Möbelhandel?}

Die Beantwortung dieser Fragen soll es der Möbelbranche ermöglichen, mit entsprechenden Optimierungen das Kundenerlebnis zu verbessern und dadurch die Kundenbindung zu erhöhen. Entlang des klassischen Kaufentscheidungsprozesses liegt der Fokus dieser Studie dabei auf den Phasen Informationssuche sowie Bewertung von Alternativen, weniger auf der Phase der eigentlichen Kaufentscheidung. Außerdem wird eher der Kauf von hochpreisigen und/oder größeren Möbel untersucht, bei dem Konsumenten tendenziell ein höheres Involvement und ein entsprechend komplexeres Kaufverhalten aufweisen (Kotler et al. 2016, S. 285 ff und 415). Nachfolgend ist der Einfachheit halber von Möbeln die Rede.

\subsection{Theoretischer Hintergrund}

\subsubsection{Die Schweizer Möbelbranche}

Der Schweizer Umsatz für Wohnungseinrichtung betrug 2017 etwa 3,85 Mrd. CHF (PwC 2017, S. 7). Werden nur Möbel betrachtet, betrug der Umsatz 2,7 Mrd. CHF (Verband Schweizer Möbelhandel und -industrie 2017). Nachdem sich die Absatzentwicklung nach Aufhebung des EUR/CHF-Mindestkurses im Jahr 2016 erstmals auf dem Vorjahresniveau stabilisierte, verzeichneten die Jahre 2017 sowie 2018 stagnierende Entwicklungen (Verband Schweizer Möbelhandel und -industrie 2019). Das ist einerseits auf die Konsumentenstimmung zurückzuführen und andererseits auf den zunehmenden Wettbewerb und den damit einhergehenden Preisdruck. Der Marktanteil importierter Möbel nimmt u. a. aufgrund von Markteintritten sowie verstärkter Präsenz ausländischer Anbieter weiter zu (z. B. IKEA, XXXLutz). Wegen der ,,verhalten positiven Konsumentenstimmung und der 
zaghaft besseren Konjunkturentwicklung, der Normalisierung des Wechselkurses ... und der immer noch robusten Bautätigkeit ..." geht der Verband der Schweizer Möbelindustrie (2019) auch für die nähere Zukunft von stagnierenden Zahlen aus.

Die Struktur der Möbelbranche zeigt eine deutliche Trennung zwischen Produktion und Handel. So ist die Möbelproduktion gekennzeichnet durch einen hohen Grad an Spezialisierung sowie Fragmentierung, und es stehen ,eine Vielzahl von Kleinstunternehmen ... wenigen großen Möbelproduzenten gegenüber" PwC (2017, S. 17). Daraus ergeben sich für Klein- und Kleinstunternehmen ein höherer Diversifikationsdruck sowie eine geringere Marktmacht. In der Schweiz gibt es ungefähr 50 Möbelherstellerbetriebe, aber die Mehrzahl der verkauften Möbel stammt aus dem Ausland (Verband Schweizer Möbelhandel und -industrie 2019). Auf der Handelsseite sind zurzeit noch vorwiegend stationär-orientierte Anbieter vertreten, die zuweilen über mehr oder weniger ausgeprägte digitale Kanäle verfügen. Reine Onlineanbieter sind in der Schweiz aktuell noch wenige aktiv tätig.

Die 10 größten Möbelhändler der Schweiz halten einen Marktanteil von über $80 \%$. Gemessen am Umsatz in Mio. CHF per 2015 wird die Liste angeführt von IKEA (Mio. CHF 1030), Conforama-Gruppe (Mio. CHF 698), Möbel Pfister-Gruppe (per 2014 Mio. CHF 603), Migros (Mio. CHF 456) mit verschiedenen Marken wie Micasa und Interio sowie Coop (Mio. CHF 293) mit TopTip (seit 2018 als Livique vermarktet). Es ist ein Konsolidierungsprozess zu beobachten, der zum Abbau von Arbeitsplätzen sowie zu Produktionsverlegungen ins Ausland führt (Verband Schweizer Möbelhandel und -industrie 2019). Letzteres wird durch neue Marktteilnehmer (z. B. XXXLutz) verstärkt. Weiter zeichnet sich eine Tendenz zu den Positionierungspolen Discount vs. Luxus ab, wobei wenig profilierte Anbieter eher Marktanteile verlieren (Verband Schweizer Möbelhandel und -industrie o. J.).

Die Möbelbranche wird maßgeblich durch die Konjunktur, die Bevölkerungsentwicklung sowie zunehmend durch die Digitalisierung geprägt (PwC 2017, S. 22). Weitere Trends sind die Individualisierung, Mobilität und Urbanisierung sowie Green Awareness. Auch der Verband Schweizer Möbelhandel und -industrie (2019) betont die zunehmende Bedeutung des Online- und Versandhandels und gleichzeitig die sinkende Bedeutung des Fachhandels. Letzteres ist auch darin begründet, dass sich Kunden kaufrelevante Informationen transparent über verschiedene Kanäle beschaffen können. „Die Nutzung von Internet-Plattformen als Informations- und Kaufkanal nimmt weiter zu. ... Die Chance des stationären Handels liegt darin, den Möbelkäufern Vorteile zu bieten, die ein elektronisches Medium nicht bieten kann“, fasst der Verband Schweizer Möbelhandel und -industrie (2019, o. S.) die Situation zusammen und bestärkt damit die Relevanz der vorliegenden Studie für den Schweizer Möbelmarkt.

\subsubsection{Konsumentenverhalten und Customer Experience}

Die Konsumentenverhaltensforschung integriert Theorien und Modelle aus unterschiedlichen wissenschaftlichen Disziplinen (für eine Übersicht, siehe Pepels, 2013). Im Rahmen dieser Studie werden die Begrifflichkeiten und Konzepte von Trommsdorff und Teichert 
(2011, S. 31) verwendet. Eine umfassende Abbildung des Konsumentenverhaltens ist vor dem Hintergrund der digitalen Transformation und den damit verbundenen, fortlaufenden Änderungen derzeit unrealistisch. Stattdessen sind regelmäßige, branchenspezifische empirische Analysen und davon abgeleitete Empfehlungen für die Praxis sinnvoller. Bis heute finden sich nur wenig empirische Daten zu den ausgewählten Erklärungsansätzen des Konsumentenverhaltens in Verbindung mit der digitalen Transformation, der Bereitstellung kaufrelevanter Informationen sowie auch Trends rund um die Informationsaufbereitung in der Möbelbranche. Die vorliegende Studie will hier einen Beitrag leisten.

Die Customer Experience ist ein Begriff aus dem übergeordneten Konzept der Kundenorientierung (vgl. beispielsweise Klaus 2015, S. xi; Smith und Milligan 2015, S. 4). Holland (2018, o. S.) interpretiert sie wie folgt: „Die Customer Experience umfasst die Gesamtheit aller Eindrücke, die ein Kunde während der gesamten Dauer einer Kundenbeziehung von einem Unternehmen erhält ... [und] umfasst sämtliche individuellen Wahrnehmungen und Interaktionen des Kunden an den verschiedenen Kontaktpunkten ...". Weiter stellt Holland (2018, o. S.) auch die Verbindung zur Kundenretention her: „Die Customer Experience stellt ein holistisches Konstrukt dar, das mehrere Prozessphasen umschließt und als vorgelagertes Konstrukt zur Kundenbindung betrachtet wird“. Dank höherer Vergleichbarkeit, geringeren Wechselkosten, vermehrter Substitute sowie zunehmenden Mitbewerbern verlieren Märkte an Attraktivität (in Anlehnung an Porter 2008). Entsprechend gaben in einer Studie 65 \% der befragten Branchenvertreter des Handels an, dass künftig Projekte zur Customer Experience wichtiger werden (Berghaus et al. 2017, S. 51). Laut einer von Accenture und Forrester (2015, zit. n. Lemon und Verhoef 2016, S. 69) durchgeführten Studie unter Führungskräften wurde das Ziel Improving the Customer Experience am häufigsten genannt, womit die Kundenorientierung zu einem zentralen Managementziel geworden ist. Demgemäß nutzen viele Schweizer Firmen entsprechende Kennzahlen (z. B. des Net Promoter Scores) ${ }^{1}$ zur Definition von strategischen Zielen (Die Schweizerische Post AG 2019, S. 7; Oberholzer und Leuthold 2018, S. 25 ff; Swisscom AG 2019, S. 35) und/oder lassen die Kundenzufriedenheit in die Vergütungsstruktur einfließen (PostFinance AG 2018, S. 33).

\subsubsection{Kaufrelevante Informationen und Kaufentscheidungsprozess}

Unter kaufrelevanten Informationen werden in dieser Studie Informationen verstanden, die die Konsumenten vor dem Möbelkauf in Erfahrung bringen wollen. Laut Frey et al. (2005, S. 366 f.) ,benötigen Käufer bei der Produktbeurteilung in der Regel zwei Informa-

\footnotetext{
${ }^{1}$ Kurz „NPS“, eine Kennzahl für die Kundenzufriedenheit bestimmt durch die Frage „Wie wahrscheinlich ist es, dass Sie das Unternehmen einem Freund oder Kollegen weiterempfehlen?“. Der NPS ergibt sich aus dem Prozentsatz der Promotoren (Antworten mit 9-10 auf einer 10er-Skala) abzüglich der Detraktoren (Antworten mit 0-6 auf einer 10er-Skala) (Oberholzer und Leuthold 2018, S. 16).
} 
tionen, nämlich Preis und Qualität“. Auch Nelson (1970, S. 312, zit. n. Leising 2010, S. 14) hält fest, dass neben dem Preis auch die Qualität zu den kaufrelevanten Informationen gehört. Informationen zur Qualität sind je nach Produkt allerdings sehr unterschiedlich verfügbar (Frey et al. 2005, S. 366). Deshalb unterscheidet Nelson 2 Kategorien des Entscheidungsverhaltens, die Suchgüter und die Erfahrungsgüter (Nelson 1970, zit. n. Fribus 2004, S. 31 sowie Leising 2010, S. 14). Suchgüter zeichnen sich dadurch aus, dass deren Qualität vor dem Kauf eingeschätzt werden kann. Bei Erfahrungsgütern ist eine Qualitätskontrolle vor dem Kauf tendenziell nicht möglich, weshalb sich Konsumenten infolgedessen auf ihre bisherige Erfahrung verlassen müssen. Laut Levin et al. (1984, zit. n. Frey et al. 2005, S. 367) führt dies dazu, dass die fehlenden Informationen durch einen ,aggregierten Wert aus vorhandenem situations-spezifischen Erfahrungswissen“ ersetzt wird. Im Zusammenhang mit Möbeln spielen neben dem Preis und der Qualität auch weitere Faktoren wie Maße, Design, Verarbeitung, Bequemlichkeit, Funktionalität etc. eine große Rolle bzw. können als kaufrelevante Information eingestuft werden (Verband der deutschen Möbelindustrie 2018).

In der Literatur findet sich eine Vielzahl an Konzepten zum Kaufentscheidungsprozess. Diese ähneln sich grundsätzlich, allerdings gibt es Unterschiede bzgl. der Anzahl, der Benennung und Beschreibung der enthaltenen Prozessschritte sowie in Bezug auf die Perspektive. Die meisten Modelle umfassen nicht nur die Kaufentscheidung per se, sondern auch vor- und nachgelagerte Phasen (Kotler et al. 2016; Peter und Olson 2010; Rutschmann und Belz 2014; Winters 2016), die typischerweise „Vorkauf“, „Kauf“ sowie „Nachkauf“ zugeordnet werden (Griese und Bröring 2011, S. 37). Der Prozess verläuft nicht unbedingt sequenziell. Stattdessen werden Phasen bzw. Schritte abgebrochen, wiederholt, gänzlich ausgelassen oder die aktuelle Phase überlappt sich mit der vor- oder nachgängigen (Griese und Bröring 2011, S. 67; Kotler et al. 2016, S. 298). Der hier skizzierte Prozess umfasst die Schritte Bedürfnis, Informationen, Alternativen, Kaufentscheidung und Bewertung der Kaufentscheidung. Nachfolgend werden die einzelnen Schritte erläutert.

Ausgelöst wird der Kaufentscheidungsprozess von einem Bedürfnis, d. h. dem Gefühl eines Mangels, verbunden mit dem Wunsch, diesen zu befriedigen (Kotler 2011, zit. n. Groissberger 2014). Mit anderen Worten entsteht Bedarf, ,wenn einem Bedürfnis ... Kaufkraft gewidmet wird, d. h. wenn der Mensch nach dem Empfinden des Bedürfnisses den nächsten Schritt tut und sich entschließt, ... dafür Kaufkraft herzugeben“ (Fischbach und Wollenberg 2014, S. 17).

In Bezug auf Möbel gibt es meist eine Vielzahl an Informationen, die Kunden vor dem Kauf in Erfahrung bringen (möchten) und die entsprechend kaufrelevant sind. Diese Informationen sind zumeist kostenlos und relativ transparent über verschiedene Kanäle zugänglich. Bei Möbeln handelt es sich in der Tendenz um sogenannte Suchgüter, d. h., es ist möglich, die Qualität der Möbel vor dem Kauf einzuschätzen, was Konsumenten in der Regel auch tun (Aemmer et al. 2019, S. 67 ff.). Zusammengefasst werden bei der Informationssuche „die aus der Bedürfniserkennung resultierenden Absichten weiter präzisiert“ (Laesser 2004, S. 21). 
Auf Basis der in Erfahrung gebrachten Informationen werden beim Kauf von Möbeln Alternativen gebildet und bewertet. Kotler et al. (2007, S. 298, zit. n. Groissberger 2013, S. 25) fokussieren stark darauf, dass verfügbare Alternativen rational gewählt werden, d. h. zuweilen bewusst dasjenige Produkt gewählt wird, das den größten Nutzwert darstellt (vgl. dazu auch von Nitzsch 2006, S. 194 ff.). Andere Modelle heben affektive ${ }^{2}$ Aspekte hervor (Laesser 2004, S. 16). In Bezug auf die Bildung von Alternativen bezeichnet von Nitzsch (2006, S. 93 f.) Menschen als ,kognitive Geizhälse“, die sich gerne an Heuristiken orientieren, v. a. am Naheliegenden (Verankerungsheuristik) und Leichtvorstellbaren (Verfügbarkeitsheuristik). Das bedeutet, dass es potenziell ,in Wirklichkeit ... noch viel bessere Alternativen“" gibt, ,,auf die der Entscheider in der Situation nicht kommt" (von Nitzsch 2006, S. 94). Eine kundenzentrierte Informationsbereitstellung sollte den Konsumenten diesen Prozessschritt erleichtern.

Mit der Kaufentscheidung fassen Konsumenten den Entscheid zum Abschluss, gefolgt vom effektiven Abschluss. Allerdings können Einstellungen von Dritten und unvorhergesehene situative Faktoren laut Kotler et al. (2016, S. 302) dazu führen, dass keine Kaufentscheidung und kein Kaufabschluss zustande kommen. Bezüglich der Einstellung von anderen kommt es auf die Intensität der jeweiligen Einstellung sowie das Verhältnis zur beeinflussenden Person an (Kotler et al. 2016, S. 302 f.). Ein geringfügig abschätzender Kommentar eines Arbeitskollegen zur Farbe eines Möbelstücks wird kaum gleich gewertet wie eine Rückmeldung des besten Freundes, wonach ein bestimmter Tisch nach eigener Erfahrung bedeutende Mängel aufweist. Dieses Beispiel hebt zugleich die Bedeutung von Bewertungen und Empfehlungen hervor, die einen Kauf verhindern, aber auch fördern können.

Nach dem Kaufabschluss kommt es oft zu einer Bewertung der Kaufentscheidung in der Nachkaufphase. So reduzieren Kunden vor und auch nach dem Kauf z. B. auftretende kognitive Dissonanzen. Dabei handelt es sich um negative Gefühlszustände bzw. Widersprüche, die beispielsweise bei unvereinbaren Kenntnissen, Annahmen und Wünschen empfunden werden (Raab et al. 2010, S. 42). Konkret können Kunden z. B. kognitive Dissonanzen empfinden, wenn sie nach dem Kauf eines Sofas feststellen, dass dieses von Käufern sehr schlecht bewertet wurde. Die Reduktion der Dissonanz erfolgt u. a. entweder über Vermeidung oder Leugnung von Informationen, der Änderung von Einstellungen oder Verhaltensweisen sowie über die selektive, bestätigende Beschaffung und Interpretation von Informationen (Maier 2018). Die Nachkaufdissonanz (Schuchard-Ficher 1979, S. 4) tritt besonders bei teureren Produkten auf, mit denen sich Konsumenten stärker beschäftigen und dabei intensiv die verschiedenen Vor- und Nachteile der verfügbaren Alternativen abwägen (vgl. auch die Ausführungen zu Involvement). Dies entspricht auch einer Eigenschaft reifer Märkte, wie beispielsweise dem Schweizer Möbelmarkt, in denen

\footnotetext{
${ }^{2}$ Affektive Entscheidungsmodelle: „Vertreter dieses Ansatzes gehen davon aus, dass Einstellungen ..., Überzeugungen, Wertvorstellungen, Risikowahrnehmung, Involvement, Persönlichkeit usw. zu den hauptsächlichen Treibern des Verhaltens gehören“ (Laesser 2004, S. 16).
} 
meist viele gleichwertige Produkte verfügbar sind, was zu einer Entscheidungsüberlastung sowie des Gefühls des Bedauerns führen kann (Gelbrich et al. 2018, S. 62).

Ergänzend zum allgemeinen Kaufentscheidungsprozess gibt es einige Besonderheiten beim Kauf von Möbeln. Kotler et al. (2016, S. 415) differenzieren Güter auf Basis des zugrunde liegenden Kaufverhaltens. Demnach gibt es Güter des täglichen Gebrauchs, Sonderprodukte und Spezialitäten, unbekannte oder unerwünschte Güter sowie Suchgüter, wobei Kotler Möbel als Beispiel für Suchgüter aufführt.

Im Kaufentscheidungsprozess gibt es zudem Unterschiede in Abhängigkeit des sogenannten Involvements sowie der Unterschiede zwischen Marken- bzw. Produkteigenschaften. Dabei kann das Involvement mit dem Grad der Beteiligung durch Konsumenten gleichgesetzt werden (Kirchgeorg 2018). Traylor (1983, S. 75) definiert das Produktinvolvement als ,recognition that certain product classes may be more or less central to an individual's life, attitudes about self, sense of identity, and relationship to the rest of the world“. Demnach tätigen Konsumenten den Kauf von Gütern und Dienstleistungen mit höherer Beteiligung, die für ihr Leben von größerer Bedeutung sind.

Der Ansatz Impulse online/offline, Research online, purchase offline (IOROPO; Friedrich und Diekmann 2016) beschreibt auf Basis des ROPO-Effekts ${ }^{3}$ idealtypische Wege, wie Konsumenten während der Phasen Impuls, Research, Kauf und Service (Nachkauf) zwischen On- und Offlinekanälen wechseln. Anschließend erfolgt die Research-Phase online, wonach der Kauf offline abgeschlossen wird. Das sind Tendenzen, ein Möbelkauf kann auch über andere Kombinationen stattfinden. Allerdings betont dieser Ansatz die Wichtigkeit, die dem stationären Handel in der Möbelbranche nach wie vor zukommt.

Diese Studie vereint verschiedene Modelle zum Kaufentscheidungsprozess. Die Basis bildet das Fünf-Phasen-Modell nach Kotler et al. (2016, S. 287). Dieses Modell wird mit den Einflussfaktoren nach Trommsdorff und Teichert (2011, S. 31) erweitert. Außerdem werden iterativere Teilprozesse innerhalb des Gesamtprozesses und in einer unbestimmten Abfolge angenommen. Dies erscheint im Zuge der digitalen Transformation als sinnvolle Ergänzung zu traditionellen Prozessmodellen (Rutschmann und Belz 2014 und - wenn auch nicht direkt im Kontext der digitalen Transformation - stellenweise Laesser 2004, S. 21). So sind Kunden heute ständig vernetzt, haben laufend sowie praktisch überall Zugang zu Informationen und stehen gleichzeitig einem wachsenden Angebot (u. a. von Onlineanbietern) gegenüber. Aus diesem Grund wird am Ende dieses Kapitels ein kombiniertes Modell hergeleitet, das die Basis bildet für die vorliegende Analyse zu den Erfolgsfaktoren der Bereitstellung kaufrelevanter Informationen im Schweizer Möbelhandel.

Die digitale Transformation bedingt neue Verhaltensmuster. Diese neuen Verhaltensmuster werden von den erwähnten Modellen allerdings noch zu wenig berücksichtigt. Rutschmann und Belz (2014) gehen von neuen Kaufmustern aus, die sich an den Polen

\footnotetext{
${ }^{3}$ ROPO: Kurz für „Research Online Purchase Offline“, ein Trend ,in purchasing behaviour, whereby a consumer searches the Internet for information ... and then purchases the item in a physical store" (Zorraquino.com o. J.). Verwandte Begriffe sind „Showrooming“ (Käufeinnen und Käufer besuchen den physischen Laden und kaufen dann online) bzw. „Webrooming“ (das exakte Gegenteil) (Khan 2018).
} 
Gewohnheit und Opportunismus orientieren. Konsumenten haben vermehrt nicht mehr nur Fernziele (z. B. ,den passenden Tisch für die Küche“) vor Augen, sondern auch Nahziele (z. B. „die Recherche nach dem Tisch macht Spaß“). Somit wird der generische Fünf-Schritt-Prozess in viele weitere, kleinere Teilschritte unterteilt, entlang denen die Kunden über Instant Gratification, also der sofortigen Belohnung (Patel 2014), geführt werden sollten. So sollten Informationen nicht nur vorhanden, sondern so aufbereitet sein, dass die Recherche erleichtert wird und bestenfalls Spaß bereitet bzw. Belohnungen enthält (Martineau 1958 und Tiu Wright et al. 2006, zit. n. Zhang et al. 2018, S. 189).

Auf Basis des Fünf-Schritt-Prozesses sowie des IOROPO-Ansatzes (Friedrich und Diekmann 2016) und des komplexen Kaufverhaltens nach Kotler et al. (2016), Rutschmann und Belz (2014) und Erkenntnissen zu Informationsquellen von Aemmer et al. (2019) ergibt sich ein kombiniertes Modell (Abb. 16.1). Dieses berücksichtigt den potenziell iterativen Prozessverlauf und betont die Wichtigkeit der Konversion sowie der Kundenzufriedenheit entlang des Prozesses (Aemmer et al. 2019, S. 46 f. und 89 f.). Dem Modell können zudem die wesentlichen Quellen der Informationsbeschaffung je Prozessschritt entnommen werden und es eignet sich damit gut als Basis zur Erforschung der Bereitstellung kaufrelevanter Informationen beim Möbelkauf.

\subsubsection{Forschungslücken}

Es gibt zahlreiche Studien zum Kaufentscheidungsprozess sowie zur Kundenorientierung (vgl. Klaus 2015, S. 5; Rutschmann und Belz 2014, S. 9). Grundsätzlich erscheinen jedoch die Auswirkungen der digitalen Transformation nach wie vor zu wenig erforscht bzw. bedarf es vor dem Hintergrund des rasanten, bedeutenden, nicht absehbaren und kontinuierlichen Wandels laufend neuer Erkenntnisse für die Wissenschaft und die Praxis. Der Fokus

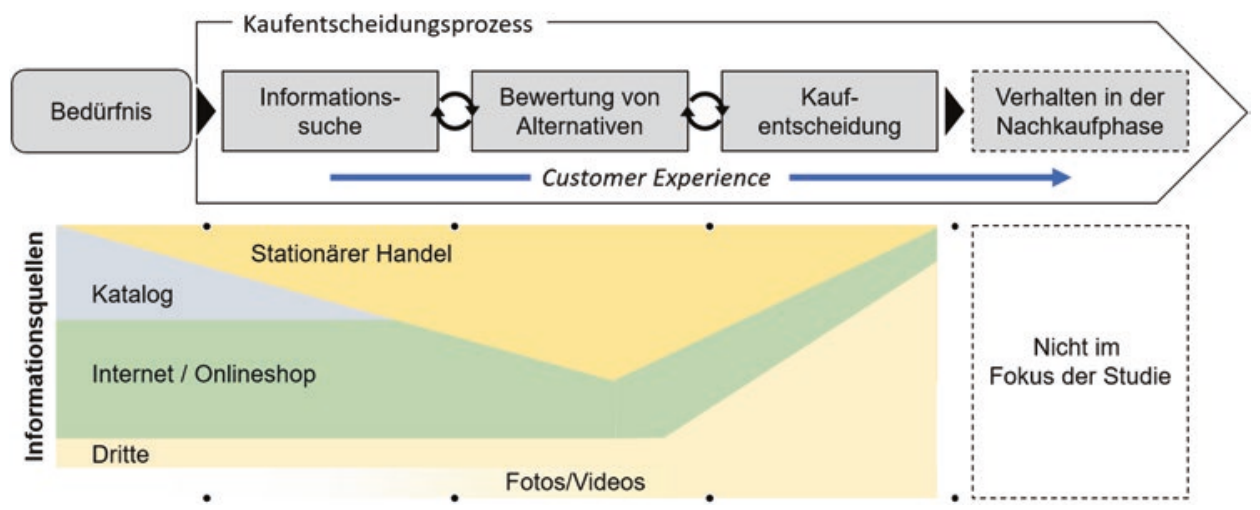

Abb. 16.1 Kombinierter Kaufentscheidungsprozess. (Quelle: eigene Darstellung in Anlehnung an Friedrich und Diekmann 2016; Kotler et al. 2016; Rutschmann und Belz 2014; Aemmer et al. 2019) 
dieser Studie liegt insbesondere auf den ersten Phasen des Kaufentscheidungsprozesses und dabei auf neuartigen Verhaltensweisen bezüglich der Inspiration, Informationsbereitstellung, -beschaffung und -bewertung über die jeweiligen Kanäle. Dadurch soll die Forschungslücke zu den Auswirkungen der digitalen Transformation auf das Konsumentenverhalten bzw. konkret auf den Umgang mit kaufrelevanten Informationen teilweise geschlossen werden. Es werden folgende Bereiche untersucht:

- Herausforderungen beim Möbelkauf

- Inspiration

- Kaufrelevante Informationen

- Kanäle (Channels) und Kundenlenkung

Die festgestellten Forschungslücken mit Bezug zur digitalen Transformation haben direkte Auswirkungen im Bereich des Channel-Managements. Dies rührt u. a. daher, dass Kanäle die Gefäße für kaufrelevante Informationen darstellen und letztere einen maßgeblichen Einfluss auf das Kundenverhalten beim Möbelkauf haben. Besonderes Augenmerk dieser Studie liegt auf dem physischen Kanal, verbunden mit der wachsenden Bedeutung digitaler Kanäle. Hierzu stehen noch wenig empirische Daten zur Verfügung. Die Praxis soll dieser Forschung konkrete Handlungsempfehlungen entnehmen können, um Herausforderungen des (Omni-)Channel-Managements zu adressieren.

\subsection{Empirische Untersuchung (Forschungsdesign)}

Ziel der Möbelhändler sollte sein, kaufrelevante Informationen entlang des Kaufentscheidungsprozesses so bereitzustellen, dass Kunden sich für ein Möbelstück aus dem eigenen Sortiment entscheiden. Dafür ist es weniger wichtig, in welchem Kanal der Kaufentscheid gefällt wird, als vielmehr, dass die Kunden aufgrund des gebotenen Kauferlebnisses gebunden werden. Im Zentrum der in den nachfolgenden Kapiteln beschriebenen Empirie stand die Frage, was die Erfolgsfaktoren für eine kundenzentrierte Bereitstellung von kaufrelevanten Informationen im Schweizer Möbelhandel sind. Für die Untersuchungen wurde ein qualitatives Forschungsdesign mit semiquantitativen Elementen gewählt. Erkenntnisse sollten dabei aus ethnografischen Beobachtungen, Leitfadeninterviews mit Kunden und ausgewählten Experten sowie einer branchenübergreifenden Analyse von Good Practices oder Leading Practices gewonnen werden.

Die theoretische Basis bildete ein Modell zum Kaufentscheidungsprozess, in dem Kunden, ausgehend von einer Inspiration bzw. einem Bedürfnis, über die Informationssuche sowie die Bewertung von Alternativen zur Kaufentscheidung und danach in die Nachkaufphase gelangen. Der Fokus lag bei der Erarbeitung der Customer Journey auf den Phasen Informationssuche und Bewertung von Alternativen, da diese beiden Phasen nur schwer zu trennen sind und Kunden oft iterativ zwischen beiden Phasen hin und her wechseln (Aemmer et al. 2019). Zudem werden auch für die Bewertung von Alternativen immer wieder 
Informationen herangezogen. Wie in Abschn. 16.4 dargelegt, wurde im Verlauf der Erhebung klar, dass auch andere Phasen des Modells berücksichtigt werden sollten. Dies gilt insbesondere für die Phase Inspiration, da auch in dieser Phase eine kundenzentrierte Bereitstellung von Information für die Kundenbindung und damit für den langfristigen Erfolg der Möbelhändler kritisch ist. Deshalb wurden zusätzlich auch Erfolgsfaktoren für die Bereitstellung von Informationen in dieser Phase identifiziert (Abb. 16.2).

Die für die Informationsbeschaffung genutzten Kanäle in der Customer Journey wurden aus Erkenntnissen einer früheren Studie abgeleitet und basieren auf der Annahme, dass sich ein Großteil der Kunden zu Beginn des Kaufentscheidungsprozesses vor dem Besuch das stationären Handels online informieren (Aemmer et al. 2019). Dass Kunden vermehrt vorinformiert in die Möbelgeschäfte kommen, wird auch von den Möbelhändlern selbst gestützt und bestätigt. So wird von einem Anteil von 50-80 \% der Kunden ausgegangen, die sich vor dem Besuch der Filiale vorgängig über digitale Kanäle informiert haben (Felber 2018). Auf der anderen Seite schätzen viele Kunden den stationären Handel beim Einkauf von Möbeln mit hohem Involvement weiterhin als wichtigen bzw. wichtigsten Kanal (vgl. Aemmer et al. 2019, S. 105; Verband der deutschen Möbelindustrie 2018).

Für den studienrelevanten Ausschnitt der Customer Journey wurde davon ausgegangen, dass Kunden aufgrund von fehlenden Informationen auf digitalen Kanälen bei Möbeln aktuell zu einem Kanalwechsel gezwungen sind, konkret zum Besuch des stationären Handels. In Bezug auf digitale Kanäle wurde deshalb folgende Teilfrage untersucht:

Teilfrage I (Information online): Wie kann das Fällen der Kaufentscheidung online bzw. auf digitalen Kanälen durch die Bereitstellung optimierter oder zusätzlicher Informationen erleichtert werden?

Für die Studie wurde angenommen, dass trotz weiterem Zuwachs an Onlineeinkäufen der stationäre Handel beim Möbelkauf mit hohem Involvement eine entscheidende Bedeutung beibehalten wird. Aus diesem Grund stellt bei einem typischen Möbelkauf der Übergang

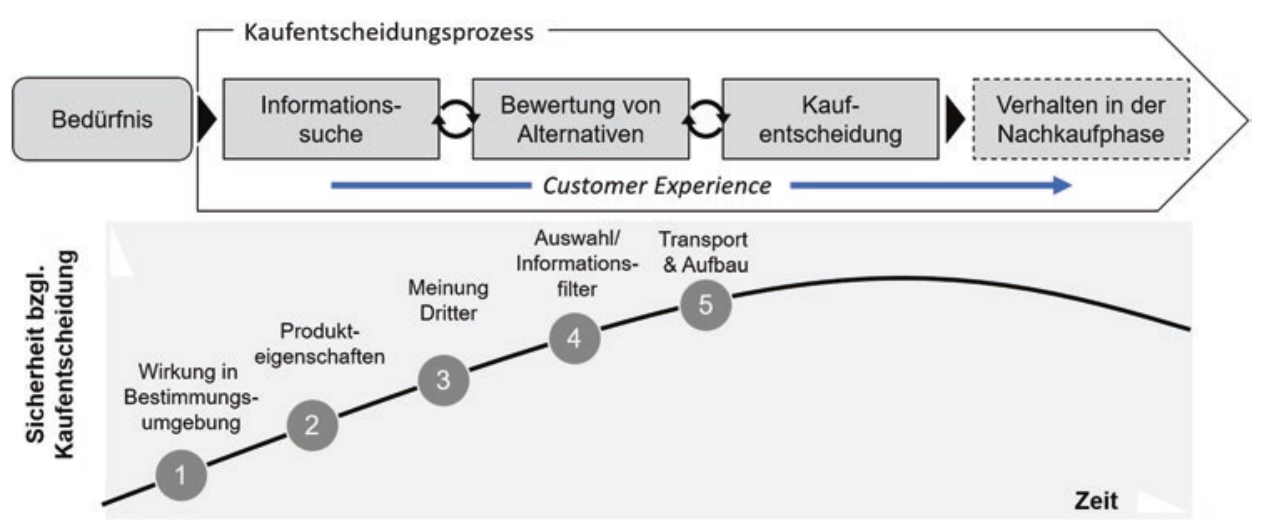

Abb. 16.2 Konzept und Methodik der Studie. (Quelle: eigene Darstellung) 
von der Informationssuche online bzw. über digitale Kanäle in den stationären Handel einen wichtigen Bestandteil des Kaufentscheidungsprozesses dar. Eine entscheidende Schwelle ist es, die Kunden mit entsprechenden Informationen und Angeboten anzuregen, den Weg in die Möbelfiliale tatsächlich auf sich zu nehmen. Aufgrund der hohen Relevanz des Übergangs wurde dieser in einer Teilfrage untersucht:

Teilfrage II (Gestaltung Übergang): Wie sollten Informationen bereitgestellt werden, damit Kunden optimal beim Kanalwechsel in den stationären Handel unterstützt sowie dafür angeregt werden können?

Im letzten Abschnitt der Customer Journey sollten Kunden den Kanalwechsel von online zum stationären Handel möglichst einfach und mit den notwendigen oder gewünschten Informationen vollziehen können. Dies wurde in einer weiteren Teilfrage analysiert:

Teilfrage III (Information stationär): Wie sollten welche Informationen im stationären Handel bereitgestellt werden, damit das Kundenerlebnis im stationären Handel nahtlos an die Onlinerecherche anknüpfen kann?

Die Konkretisierung der übergeordneten Forschungsfrage mithilfe der aufgeführten 3 Teilfragen diente dazu, die empirische Datenerhebung zu strukturieren.

\subsubsection{Ethnografische Beobachtungen}

Der Einstieg in das Forschungsfeld erfolgte mit ethnografischen Beobachtungen in der Filiale von Möbel ITURE in Bern (Schulz 2014, S. 225). Es wurde versucht, die hypothetische Customer Journey durch Beobachtungen des Verhaltens von Kunden im stationären Handel zu validieren. Konkret wurde eingeschätzt, wie zielstrebig Kunden Möbelstücke ansteuerten. Dies galt als Indiz dafür, ob Kunden bereits vor dem Besuch der Filiale Informationen zum jeweiligen Wunschobjekt gesammelt hatten und es so eine Vorselektion gab. Wo diese vermutete Vorinformation beschafft wurde, konnte jedoch nicht beobachtet werden. Weiter wurde beobachtet, welche kaufrelevanten Informationen den Kunden in der Filiale zur Verfügung gestellt und wie sie diese wahrnahmen bzw. nutzten. Schließlich wurde die Bedeutung der Beratung bzw. die Interaktion zwischen Kundenberatern und Kunden beim Kaufentscheidungsprozess analysiert, da die Beratung eine wichtige zusätzliche Informationsquelle im stationären Handel darstellt.

\subsubsection{Leitfadeninterviews}

Es wurden 13 Kunden in den Möbelfilialen von Möbel ITURE sowie von FURNIT interviewt (Tab. 16.1). Zusätzlich wurden 5 Experten aus der Möbelbranche mit Bezug zu digitaler Transformation und Channel-Management befragt. Die Hauptstruktur für den In- 
Tab. 16.1 Charakterisierung der interviewten Experten

\begin{tabular}{l|l}
\hline Expertenkennung & Expertise \\
\hline Experte 1 & Channel-Management \\
\hline Experte 2 & eCommerce \\
\hline Experte 3 & Digital Channel Management \\
\hline Experte 4 & Digitale Transformation \\
\hline Experte 5 & Branchenvertreter
\end{tabular}

Die Experten wurden anonymisiert, um Neutralität zu gewährleisten.

terviewleitfaden wurde an den 3 Teilfragen aus der Customer Journey angelehnt. Es wurden zusätzliche Fragen definiert, die teilweise über die Customer Journey hinausgingen. So wurde beispielsweise im Rahmen des Warm-ups die Frage gestellt, wo sich die Kunden die Inspiration für den Möbelkauf herholten. Danach wurde in einem 1. Block abgefragt, was als kaufrelevante Informationen angesehen wird. Zusätzlich wurden spezifische Fragen zur Nutzung von Kanälen und Technologien gestellt. Vor der Anwendung mit den Kunden wurde der Leitfaden einem Pre-Test unterzogen. Um geeignete Kunden für Interviews zu gewinnen, positionierten sich die Interviewer jeweils in den Filialen von FURNIT und Möbel ITURE in Bereichen mit Möbeln, bei denen ein hohes Involvement beim Kaufentscheidungsprozess zu vermuten war. Zudem wurden nur Kunden angesprochen, bei denen aufgrund ihres Verhaltens ein tatsächlich vorhandenes Bedürfnis vorausgesetzt werden durfte.

Im Leitfaden für die Experten wurde vermehrt nach Good Practices oder Leading Practices sowie der Perspektive der Anbietenden gefragt. Von der Strukturierung her jedoch wurden die gleichen Themenblöcke gewählt wie schon beim Leitfaden für die Interviews mit den Kunden. So konnten die Aussagen der Kunden gut mit den Erläuterungen der Experten verglichen werden. Da die Interviews mit Experten erst nach dem 1. Interviewblock mit Kunden durchgeführt wurden, konnten bereits erste Erkenntnisse einfließen und einige Punkte zur Bereitstellung kaufrelevanter Informationen aus den Interviews mit den Kunden bewusst vertieft werden (Halbmayer und Salat 2011). Um zudem den unterschiedlichen Spezialisierungen der Experten Rechnung zu tragen, wurden nicht sämtliche Fragen allen Experten gestellt. Auch der Leitfaden für die Experten wurde einem Pre-Test unterzogen.

Die Interviews wurden transkribiert und codiert. Insgesamt wurden über 830 Aussagen codiert. Die angewandten Kategorien wurden deduktiv von der verknüpften Theorie bzw. vom Ausschnitt der hypothetischen Customer Journey abgeleitet und entsprechen daher der Struktur der Interviewleitfäden (Vogt und Werner 2014, S. 55). Insgesamt wurden für diese First-Cycle-Codierung 4 Code-Familien mit insgesamt 41 spezifischeren Codes angewendet. Zusätzlich wurden auf Basis der 1. Analyse des Datenmaterials und anhand einer Verdichtung bzw. Verallgemeinerung der Aussagen weitere 9 Codier-Kategorien induktiv definiert (Vogt und Werner 2014, S. 58 ff.). 


\subsubsection{Analyse Good Practices oder Leading Practices}

In den Interviews wurde ergänzend nach Beispielen aus anderen Branchen gefragt. Die zugrunde liegende Annahme war, dass trotz naheliegender Unterschiede beim Kaufentscheidungsprozess innovative Lösungen zur Verknüpfung der Kanäle insbesondere im stationären Handel auch für die Möbelbranche Anwendung finden können. Die Analyse von Good Practices bzw. Leading Practices bezieht sich auf Webseiten bzw. Onlineshops und physische Besuche des stationären Handels. Berücksichtigt wurden dabei Beispiele aus der Mode-, der Detailhandels- und der Heimelektronikbranche.

\subsection{Ergebnisse}

In diesem Kapitel werden die Ergebnisse der Erhebung zu den Erfolgsfaktoren für eine kundenzentrierte Bereitstellung von kaufrelevanten Informationen im Schweizer Möbelhandel zusammengefasst. Dafür werden Inspirationen, die Herausforderungen beim Möbelkauf, kaufrelevante Informationen und die verschiedenen Informationskanäle genauer analysiert.

\subsubsection{Inspiration}

Die Inspiration war als Phase des Kaufentscheidungsprozesse vor der empirischen Datenerhebung nicht im ursprünglichen Fokus der Studie. Jedoch wurde sie als Eingangspunkt im Prozess in den Interviews von Kunden wie auch Experten immer wieder angesprochen. So wurden im 2. Auswertungsdurchlauf ganze 46 Aussagen mit dem Code „Inspiration“ versehen. Nachfolgend die Ergebnisse bezüglich verschiedener Inspirationsquellen.

\subsubsection{Inspiration aus Katalogen und Broschüren}

Einige Kunden gaben an, sich klassisch in Katalogen und Broschüren nach neuen Einrichtungsideen umzusehen. Typisch für diese Gruppe war folgende Aussage: „,.. und um Ideen zu holen, ist er [= der Katalog] wirklich cool. Aber ich würde darin nie ein Produkt suchen gehen“. In der Altersgruppe 40+ ist die Bedeutung dieses Kanals noch relativ hoch. Jüngere Kunden gaben dagegen an, Kataloge nur sporadisch und eher zufällig durchzublättern, diese jedoch nicht bewusst als Inspirationsquelle zu nutzen.

Die Experten waren bezüglich der Bedeutung von Katalogen und Broschüren geteilter Meinung. Der Branchenvertreter Experte 5 wies ihnen eine hohe Bedeutung zu. Anders sieht das der eCommerce-Experte Experte 2: „Ich glaube das spielt überhaupt keine Rolle, das ist überholt. ... Kataloge hatten eine Rolle im Möbelkauf, weil auf der Fläche in den Filialen nicht alles verfügbar war. Da konnte man den Kunden zeigen, was es sonst noch gibt. Ich glaube es wäre sträflich, wenn man heute an diesen Katalogen festhält ...". Er 
relativierte diese Aussage nach einer Nachfrage und präzisierte, dass es sicher noch eine entsprechende Zielgruppe gebe, aber auch viele der älteren Generation (,Silver Surfers“) mittlerweile aktiv online seien. Der Channel-Manager von FURNIT sah auch einen Wandel, der jedoch nicht abgeschlossen ist: „Es gehört zum FURNIT-Konzept dazu, dass man halt den Katalog hat. ... Natürlich hat er immer noch seine Berechtigung, daher macht man ihn auch logischerweise. Aber ich glaube, heutzutage gibt es andere Mittel, die eher im Onlinebereich anzusiedeln sind, die mehr Einfluss haben“. Der digitale Transformationsspezialist Experte 1 differenzierte weiter: „Wohldosiert [spielt der Katalog] eine sehr wichtige Rolle. Das heißt konkret, noch seltener Kataloge rausbringen, die wertig sind, nahe am Buch, die man auch ... - weil man es schön findet - in der Wohnung hat, um sich sozusagen kontinuierlich mit diesen Themen zu beschäftigen. Wenn man es schafft, über derartige physische Sendungen die Leute am Thema interessiert zu halten, dann gewinnt man viel mehr als über einen Newsletter". Die Aussagen aus den Interviews zeigen, dass aktuell Kataloge und Broschüren zumindest von einem Teil der Kunden als Inspirationsquelle genutzt und geschätzt werden; dementsprechend werden Möbelanbietende wahrscheinlich weiterhin daran festhalten.

\subsubsection{Inspiration aus dem stationären Handel}

Viele Kunden lassen sich direkt im stationären Handel inspirieren. Ein Paar, das ohne konkrete Kaufabsicht in der FURNIT-Filiale war, antwortete auf die Frage zum Grund ihres Besuches: „,Go schnöiggä‘ [stöbern], wie man es so schön auf Berndeutsch sagt“. Zu erwähnen ist, dass viele Kunden, die den Katalog angaben, auch die Filiale als Inspirationsquelle nannten.

\subsubsection{Inspiration online}

Tendenziell jüngere Kunden gaben an, sich online inspirieren zu lassen. Dies können einerseits Onlineshops oder auch die digitale Version eines Katalogs sein. Dazu erläuterte der eCommerce Experte 2: „Also zuerst kriege ich eine Inspiration. Die kriege ich ja heute oft aus sozialen Medien oder aus irgendwelchen Websites, die man angezeigt bekommt ...". Als typische Social-Media-Kanäle wurden Facebook und Pinterest erwähnt. Passend hierzu erklärte Experte 3: „Was sicher hinzugekommen ist, sind die ganzen sozialen Kanäle, die jetzt gerade im Interior-Bereich halt schon auch eine wichtige Rolle spielen“. FURNIT verzeichnete Anfang Juni 2019 über 72.000 Besucher pro Monat über Pinterest, Möbel ITURE erreichte Anfang Juni über 35.000 Besuchern pro Monat. Die Experten im Bereich eCommerce, digitale Transformation und Channel-Management haben die wachsende Relevanz dieser Kanäle unterstrichen. Gleichzeitig wurde auch auf die damit verbundenen Herausforderungen hingewiesen. Experte 2 führte beispielsweise aus: „Der Händler kann viel direkter und viel einfacher an seine Zielgruppen und auch Interessenten erreichen, die heute noch nicht zu seiner Zielgruppe zählen. ... Die Herausforderung dabei ist aber, dass man im Online- und Mobile-Auftritt dann auch wirklich Inspiration schafft, dass man den Kunden dort abholt und dass man wirklich mit ihm interagiert". Hinweise darauf, wie Anbietende diesen Kanal erfolgreich nutzen und kauf- 
relevante Informationen kundenzentriert bereitstellen können, werden in nachfolgendem Kapitel gegeben.

\subsubsection{Inspirierende Information - Storytelling}

Es lässt sich kritisch hinterfragen, was Inspiration mit der Bereitstellung kaufrelevanter Informationen zu tun hat. Mit der Inspiration wird der Kaufentscheidungsprozess ausgelöst und es entsteht überhaupt erst ein Bedürfnis bei Kunden. Deshalb können Geschichten, die rund um Möbel erzählt werden, ebenfalls als kaufrelevante Information eingestuft werden. Experte 3 erklärte dies folgendermaßen: „Inspiration steht am Anfang von allem. ... Dass sich die Leute ein besseres Wohnzimmer - oder welchen Raum sie auch immer einrichten wollen -, dass sie sich das mit unserem Range, mit unseren Produkten vorstellen können. ... Also so die Bedürfnisse wecken. ,Ich kann gewisse Sachen meines Lebens besser machen mit Möbel von uns“". Aus nachfolgender Aussage von Experte 4 lässt sich ableiten, wieso bei Möbeln gerade die inspirierenden Informationen durchaus als kaufrelevant bezeichnet werden können: „Gerade, wenn es um Design geht, geht es auch viel um Geschichten, was die Inspiration angeht. Wer die Person ist, die sich das ausgedacht hat. Da wird ja dann das Narrativ deutlich wichtiger, als die bloßen Spezifikationen wie Größe und Höhe, die eigentlich sekundär sind“. Experte 1 wiederum führte zur Konfiguration der verschiedenen Touchpoints aus: „Dann finde ich, man sollte davon wegkommen, von Informationen zu sprechen, die man am Touchpoints rüberbringen will. Sondern eigentlich sollte man eher von Werten sprechen, die man kreieren will ...". Was mit solchen Geschichten und Werten bzw. dem im Titel dieses Kapitels aufgeführten Storytelling gemeint sein könnte, konkretisierte Experte 2: „Mein Lieblings-Webshop ist Mister Porter ... ein Fashionshop. Was den ausmacht, ist, dass die eine unglaubliche Vielzahl an inspirierendem redaktionellem Content haben. Die bieten Newsletter an, da sind bekannte Schauspieler oder irgendwelche Architekten, ... zum Beispiel Jude Law, der erzählt, wo er am liebsten reist und was er dann trägt. Das ist aber redaktionell so gut aufbereitet, dass ich eigentlich in jeden Newsletter reinklicke und auch auf der Seite immer etwas finde“. Es geht also darum, interessanten Inhalt (oben „Content“) für Kunden zusammenzustellen. Dieser Inhalt hat nicht unbedingt direkt mit dem Produkt selbst zu tun, sondern setzt Produkte in einen Kontext. Dies geschieht auch mit der Inszenierung von Möbeln in einem Katalog. Beim Storytelling werden die Bilder mit weiteren Dimensionen erweitert. Der Mensch mit seinen Bedürfnissen oder die Geschichten rund um die Produkte werden in den Vordergrund gestellt und es wird mit, statt über Produkte kommuniziert.

Geschichten gut zu erzählen, ist eine eigene Marketingherausforderung. Treffend formulierte es Experte 4: „Mir bringt keine geile eigene Website was, mit einem super Narrativ und einer super Story, wenn die Leute nach meinen Produkten nur auf der Plattform suchen“. Wie Experte 1 erklärte, reiche es aber nicht aus, die richtigen Plattformen zu finden. So sei es ,... ganz wichtig, dass man immer schaut, warum eine Person auf eine Plattform geht, was will sie dort. Zum Beispiel erwarte ich etwas anderes auf Facebook und Instagram, als wenn ich auf LinkedIn oder Tumblr gehe. Und ich glaube, die Art und 
Weise der Ansprache, aber auch die Botschaft oder die Information selbst, sollten einen Bezug haben zu dem, was ich auch auf der Plattform suche“. Um inspirierende Geschichten zu erzählen, sollten also zunächst die richtigen Plattformen identifiziert werden, nämlich solche, wo die eigene Zielgruppe sich aufhält bzw. nach den eigenen Produkten gesucht werden könnte. Die Geschichten wiederum sollten so erzählt werden, dass sie mit dem Zweck bzw. den Inhalten der Plattform passen.

\subsubsection{Herausforderungen beim Möbelkauf}

Auf die Frage zu Herausforderungen beim Möbelkauf antwortete eine Kundin bei Möbel ITURE: „Dass ich wirklich sicher sein kann, dass es das jetzt ist und es nicht noch etwas Besseres gibt“". Es fällt Kunden demnach schwer, die Unsicherheit bezüglich des Kaufentscheids so weit zu reduzieren, dass sie diesen tatsächlich fällen können. Die aufgrund fehlender Informationen vorhandene Unsicherheit bezüglich der Wirkung der Möbel in der Bestimmungsumgebung sowie bezüglich weiterer Produktmerkmale wird in den ersten 2 Unterkapiteln beleuchtet. Danach wird auf die Bedeutung der Meinung von Dritten eingegangen. Im 4. Unterkapitel werden die Herausforderungen aufgrund zu vieler Informationen bzw. einer zu großen Auswahl beschrieben. Weiter wird erklärt, dass der Transport der Möbel sowie der Weg in die Filiale ebenfalls herausfordernd sein können.

\subsubsection{Unsicherheit bezüglich Wirkung in der Bestimmungsumgebung}

Die Ergebnisse aus den Interviews zeigen, dass es Kunden schwierig finden, ausreichend Sicherheit darüber zu gewinnen, ob das Möbelstück zuhause in die Bestimmungsumgebung passt. Dies unterstreicht das hohe Involvement beim Kaufentscheidungsprozess. Bei einer Zuordnung der Herausforderungen zu den Phasen des Kaufentscheidungsprozesses wird klar, dass die Problematik nicht erst beim Entscheid selbst relevant wird. Die Informationen bezüglich der Wirkung des Möbels in der Bestimmungsumgebung sind kaufrelevant und werden entsprechend im Rahmen einer Informationssuche zusammengetragen. Danach werden diese Informationen benötigt, um eine Bewertung der Alternativen vorzunehmen. Für die Beantwortung der Forschungsfrage gilt es daher zu eruieren, wie diese Informationen zielführend bereitgestellt werden können.

\subsubsection{Unsicherheit bezüglich Qualität und weiterer Produktmerkmale}

Von Kunden gemachte Aussagen zu Produktmerkmalen, z. B. der Qualität, sind mit der Unsicherheit in der Nachkaufphase verbunden. In diesem Zusammenhang gaben Kunden an, sich vor Ort im stationären Handel ein Bild des entsprechenden Möbelstücks machen zu wollen. Ein Kunde erklärte dazu: „Ich muss es ... anschauen können. Ich denke, über das Internet wird es ziemlich schwierig, dem Kunden das zu bieten, dass er weiß, dass es gute Qualität ist. Weil auch, wenn es auf dem Foto gut aussieht, ... also ich vertraue auch Fotos im Internet nicht. Ich denke, ich muss die Sachen erst selbst anschauen, bevor ich sie kaufe. Gerade auch teurere Möbel“. Ähnliche Aussagen machten Kunden bezüglich Er- 
höhung der Sicherheit hinsichtlich weiteren Produktmerkmalen. Je nach Möbelkategorie wurden von den Kunden die Überprüfung der Materialisierung, der Haptik, des Komforts, der Robustheit oder der Wirkung der Möbel im Raum als Begründung für den Gang in den stationären Handel angegeben. Dieser Mehrwert des stationären Handels wurde auch von den Experten bestätigt. Die Bedeutung der verschiedenen Kanäle wird später noch ausführlich beschrieben. Es gilt an dieser Stelle zusammenfassend festzuhalten, dass beim Möbelkauf die Herausforderung besteht, die Sicherheit zu diversen Produktmerkmalen zu erhöhen und damit die kognitive Dissonanz zu reduzieren. Aktuell gelingt dies aus Sicht eines Großteiles der Kunden in ausreichendem Maß nur im stationären Handel.

\subsubsection{Die Meinung Dritter}

Kunden gaben an, dass es herausfordernd sei, sich mit Mitentscheidenden auf eine bestimmte Alternative zu einigen. In eine ähnliche Richtung ging die Aussage einer Kundin bei FURNIT, der es wichtig war, dass auch ihre Freunde das gekaufte Möbelstück mögen. In solchen Situationen wollen Kunden die Sicherheit bezüglich einer infrage kommenden Alternative bei Mitentscheidenden erhöhen oder auch die Meinung Dritter einbeziehen, um selbst sicherer zu werden. Entsprechend äußerte sich eCommerce Experte 2: „... und ich möchte das dann eigentlich auch ganz einfach teilen können ... und sagen: , Guck mal drauf, wie findest du das, passt das? “ Der Bedarf des Einbezugs Dritter beim Möbelkauf konnte bereits in einer früheren Studie festgestellt werden (vgl. Aemmer et al. 2019, S. 102). Der Einfluss von Drittmeinungen wird zudem im Zusammenhang mit der Relevanz von Kundenbewertungen und bei der Inspiration unten diskutiert.

\subsubsection{Informationsflut und zu viel Auswahl}

Kunden bietet das Internet ein deutlich größeres Angebot als im stationären Handel. Der Channel-Management-Experte, Experte 1, hat eine Auswirkung davon folgendermaßen beschrieben: „Es ist einfach die schiere Menge. Da gibt es sicher auch Studien darüber. Ich habe das Gefühl, manchmal denkt man noch ,okay, ich könnte noch was kaufen', aber danach schaust du das Ding an und nur schon angefangen, sich damit auseinanderzusetzen, denkt man, oh nein, da hinten hat es irgendwie gefühlte fünftausend Sofas, das schaffe ich nicht'“. Tatsächlich haben die Informationsflut und die Strategien, damit umzugehen, mit insgesamt 27 codierten Aussagen einen Schwerpunkt bei den Interviews mit den Experten dargestellt. Die Gewichtung des Themas ist umso bedeutsamer, als dass die Interviewer die Informationsflut nicht direkt ansprachen bzw. keine Fragen explizit zu diesem Punkt stellten. Die entsprechende Code-Kategorie wurde erst im 2. Analysedurchlauf festgelegt. Mit 9 weiteren codierten Aussagen haben auch Kunden auf die Herausforderungen im Zusammenhang mit der großen Auswahl sowie der Informationsflut hingewiesen. Dabei besteht die Herausforderung vor allem online. Eine Möbel ITURE-Kundin führte diesbezüglich aus: „Ich denke online sind vielleicht viele Informationen vorhanden, aber die Schwierigkeit ist dort eher, sich zurechtzufinden mit den Informationen oder mit der Flut von Informationen oder an die richtigen Informationen zu kommen". Im Zuge der ethnografischen Beobachtungen ist aufgefallen, dass im stationären Handel ebenfalls eine 
große Menge an Informationen bereitgestellt wird. Die Informationsmenge wirkt jedoch für viele nicht problematisch. Die meisten Kunden erklärten, dass ihnen die Einrichtung der Filialen sehr gut gefalle. Bezeichnend dafür steht dieses Zitat eines Ehepaars: „,.. Möbel ITURE inspiriert uns jedes Mal, auch die Ausstellung. Die machen das ... sehr gut““.

\subsubsection{Der Transport nach Hause und der Weg in die Filiale}

Die Logistik des Möbelkaufs stellt für einige Kunden eine Herausforderung dar. Eine Kundin bei Möbel ITURE gab als für sie relevante Information an, ob die Möbel geliefert und aufgebaut werden können. Eine Kundin bei FURNIT erwähnte, dass sie ihr Wunschobjekt jetzt gefunden habe, es aber nicht direkt mitnehme, sondern bestellen und liefern lassen werde. Ein Pärchen begründete seinen Besuch der Möbel ITURE-Filiale folgendermaßen: „Wir wohnen in der Stadt. Das ist jetzt ein relativ großer Möbelladen und hat auch eine gewisse Fläche, wo sie Sachen ausstellen können. In anderen Läden ist es aber so, dass man auf [Stadt, ca. $20 \mathrm{~km}$ entfernt] oder so gehen muss, um sich das anzuschauen. Wir haben kein Auto, daher geht man nicht schnell zu FURNIT ein Sofa oder kleinere Sachen kaufen“. Herausfordernd ist somit für eine gewisse Kundengruppe auch der Weg zum stationären Handel. So erwähnte der Digital-Channel-Manager bei FURNIT, Experte 3: „Es geht halt in Richtung Stadt. Das sieht man bei anderen Anbietern, die auch immer mehr in die Stadt finden. Wie z. B. Zara Home oder H\&M. Ich glaube, diese Bewegung ist ziemlich offensichtlich. Da werden wir natürlich auch unser Bestes geben, um stationär noch mehr in der Stadt stattzufinden“.

\subsubsection{Kaufrelevante Informationen}

Haben Kunden sich inspirieren lassen oder ist ein effektives Bedürfnis für einen Möbelkauf vorhanden, folgen die beiden Phasen Informationssuche und Bewertung von Alternativen. Es fällt Kunden schwer, sich zu entscheiden. Die Befürchtung, dass das Möbel nicht in die Bestimmungsumgebung passt, den Mitmenschen nicht gefällt, die Qualität nicht stimmt oder irgendwo im Angebotsdschungel doch noch eine bessere Alternative zu finden wäre, schwingt stets mit. Es stellt sich deshalb die Frage, welche Informationen den Kunden helfen, einen Entscheid zu fällen. Deshalb werden in diesem Kapitel mögliche kaufrelevanten Informationen anhand der Aussagen in den Interviews gruppiert und in Zusammenhang mit den Phasen des Kaufentscheidungsprozesses gesetzt. Danach werden die von Interviewpartnern am höchsten gewichteten bzw. erwähnenswertesten Informationskategorien vorgestellt.

\subsubsection{1 Übersicht und Strukturierung}

Eine Übersicht über alle kaufrelevanten Informationen herzustellen, ist kaum möglich. So erklärte Experte 2, dass allein auf Onlineshops über 300 Einzelkriterien zusammenkämen. Die Antworten der Kunden auf die Frage, was für sie kaufrelevante Informationen darstellen, könnten jedoch darauf hindeuten, welche Informationen besonders relevant sind. 
Das Optische bzw. das Design, die Dimensionen, die Funktionalität, der Preis, die Qualität, die verwendeten Materialen sowie die Pflege davon oder der Komfort wurden immer wieder genannt. Eine besonders wichtige Rolle spielt der Preis. So haben mehrere Experten darauf hingewiesen, dass mit dem Internet bzw. der digitalen Transformation eine höhere Preistransparenz entstanden sei. Der Branchenvertreter Experte 5 äußerte dazu seine Bedenken: „Heute positionieren sich alle irgendwie über Aktionen, über noch mehr Rabatt. ... [Es] sind für mich sehr viele Möbeldetailhändler einfach sehr ähnlich. Und alle profilieren sich über den Preis“. Abgesehen vom Preis sind nicht bei jedem Möbelstück die gleichen Informationen relevant. Dies zeigt sich in der Antwort des Begleiters einer Interviewpartnerin bei FURNIT. Dieser erklärte, er möchte ,... beim Sofa vor allem einmal draufsitzen. Schauen, ob es bequem ist“ oder bei einer Kommode „... die Schublade rausziehen, nicht dass es gerade auseinanderfällt". Eine Kundin bei FURNIT hat darauf hingewiesen, dass die zur Verfügung stehende Auswahl (Modelle, Ausführungen bzw. die Individualisierbarkeit) eine relevante Information sei. Obwohl keine konkrete Frage dazu gestellt wurde, gaben viele Kunden an, dass ihnen Nachhaltigkeit wichtig sei. Weitere Stichworte waren Verfügbarkeit, Lieferzeiten, Liefer- und Aufbauservice, Rückgaberecht, Garantie und Reparaturservice. Auch die Experten haben bei der Frage nach kaufrelevanten Informationen diese Faktoren bestätigt.

Basierend auf den Interviews wurden kaufrelevante Informationen gruppiert. Experte 3 führte dazu aus: „Die Grundsachen sind sicher der Preis und die Verfügbarkeit. Das sind einmal Grundvoraussetzungen, die gegeben sein müssen, damit man überhaupt rein technisch einen Vertrag oder einen Kauf abschließen kann“. Als weiteren Schritt gab er an: „Und dann sind es natürlich all die Faktoren wie eben Farben, Materialen, Maße deiner Möbel“. Danach fügte er weiter an „Dann natürlich der Stil. Passt es überhaupt zu meiner Einrichtung? ... Das sind so all die quasi kleinen Bits and Pieces, die sich zu einem ganzen Bild zusammenfügen“. Weiter erklärte Experte 3, was aus seiner Sicht die Geschichte rund um das Produkt oder die Nachhaltigkeit förderliche Aspekte darstellen, aber eben sicher nicht Hard Selling seien. Anhand dieser Ausführungen von Experten und Kunden wird in Tab. 16.2 eine Auswahl von kaufrelevanten Informationen den Phasen des Kaufentscheidungsprozesses zugeordnet. Es ist zu beachten, dass die Auswahl der Informationen auf keinen Fall abschließend ist, sondern Anhaltspunkte für die danach beschriebenen Erfolgsfaktoren bei der Bereitstellung von Information darstellen sollen.

\subsubsection{Produktempfehlungen}

Produktempfehlungen spielen an mehreren Stellen im Kaufentscheidungsprozess eine Rolle. Einerseits erscheint es nachvollziehbar, dass Kunden ganz zu Beginn durch eine passende Empfehlung inspiriert werden können. Andererseits erleichtern bzw. verkürzen auf die eigenen Bedürfnisse zugeschnittene Produktempfehlungen den Prozess. Jedoch waren sich die Experten 2,3, und 4 einig, dass hier die Reise erst gerade begonnen habe. Experte 2 formulierte es so: ,Ich finde auch gerade Produktempfehlungen sind ein spannendes Thema. Und die kann man online ganz gut spielen, aber sie passen oft auch noch nicht so gut. Also das sind irgendwelche Recommendation Engines. Ich weiß nicht, auf 
Tab. 16.2 Strukturierung kaufrelevanter Informationen

\begin{tabular}{|c|c|c|}
\hline Mögliche Fragestellungen & Auswahl an Informationen & Phase \\
\hline Was gefällt mir? & $\begin{array}{l}\text { Geschichten inkl. Nachhaltigkeit, } \\
\text { Einrichtungsideen, Bilder, } \\
\text { Produktempfehlungen }\end{array}$ & $\begin{array}{l}\text { Inspiration } \\
\text { (Entscheid) }\end{array}$ \\
\hline $\begin{array}{l}\text { Wieviel möchte ich ausgeben und } \\
\text { welche sonstigen Erwartungen an } \\
\text { die Produktmerkmale habe ich? }\end{array}$ & \multirow{2}{*}{$\begin{array}{l}\text { Preise, Verfügbarkeit, Masse, Qualität, } \\
\text { Funktion, Farben, Design, Stil, } \\
\text { Komfort, Materialien, Pflegehinweise, } \\
\text { Widerstandsfähigkeit, } \\
\text { Produktempfehlungen }\end{array}$} & $\begin{array}{l}\text { Vorbereitung } \\
\text { Informationssuche }\end{array}$ \\
\hline $\begin{array}{l}\text { Welche Auswahl habe ich mit } \\
\text { meinen Vorbedingungen? }\end{array}$ & & Informationssuche \\
\hline $\begin{array}{l}\text { Was passt in die } \\
\text { Bestimmungsumgebung? }\end{array}$ & $\begin{array}{l}\text { Bilder von Möbeln im Kontext (online), } \\
\text { Möbel im Kontext (stationär/ } \\
\text { Augmented Reality), Beratung, } \\
\text { Produktempfehlungen }\end{array}$ & $\begin{array}{l}\text { Informationssuche/ } \\
\text { Bewertung von } \\
\text { Alternativen }\end{array}$ \\
\hline $\begin{array}{l}\text { Welche Alternative sagt mir am } \\
\text { meisten zu und was halten Dritte } \\
\text { davon? }\end{array}$ & $\begin{array}{l}\text { Preis-Leistungs-Verhältnis, Beratung, } \\
\text { Kundenbewertungen }\end{array}$ & $\begin{array}{l}\text { Bewertung von } \\
\text { Alternativen }\end{array}$ \\
\hline $\begin{array}{l}\text { Und wenn es mir doch nicht } \\
\text { gefällt? }\end{array}$ & $\begin{array}{l}\text { Rückgaberecht, Rückgabeprozess, } \\
\text { Umtauschmöglichkeit, } \\
\text { Wiederverkaufsmöglichkeit, } \\
\text { Prozessbewertungen }\end{array}$ & $\begin{array}{l}\text { Bewertung von } \\
\text { Alternativen/ } \\
\text { Entscheid }\end{array}$ \\
\hline Und wenn es kaputt geht? & $\begin{array}{l}\text { Garantie, Reparaturservice, } \\
\text { Prozessbewertungen }\end{array}$ & $\begin{array}{l}\text { Bewertung von } \\
\text { Alternativen/ } \\
\text { Entscheid }\end{array}$ \\
\hline $\begin{array}{l}\text { Wie bringe ich es nach Hause, } \\
\text { baue es zusammen und werde das } \\
\text { alte Möbel los? }\end{array}$ & $\begin{array}{l}\text { Maße, Paketgrößen, Abholorte (Click } \\
\text { and Collect), Liefer-, Aufbau-, } \\
\text { Entsorgungsservice }\end{array}$ & $\begin{array}{l}\text { Bewertung von } \\
\text { Alternativen/ } \\
\text { Entscheid }\end{array}$ \\
\hline Wie bezahle ich? & Bezahlmöglichkeiten & $\begin{array}{l}\text { (Bewertung von } \\
\text { Alternativen)/ } \\
\text { Entscheid }\end{array}$ \\
\hline
\end{tabular}

welcher Basis die da Empfehlungen auswerfen. ... Da gibt es Verbesserungspotenziale“. Experte 3 meinte, dass zukünftig sicherlich mithilfe von Algorithmen von großen TechFirmen oder auch von FURNIT selbst Produktempfehlungen aufgrund von Kundendaten geschaltet würden. Experte 4 sprach von der ,theoretischen Möglichkeit, über Nutzerinformationen andere Einschätzungen $\mathrm{zu}$ Produkten $\mathrm{zu}$ bekommen. Empfehlungen $\mathrm{zu}$ bekommen, die näher an den eigenen Bedürfnissen sind“. Den Nutzen von solchen bedürfnisspezifischen, datenbasierten Produktempfehlungen sieht Experte 3 wie auch Experte 4 als Teil einer kundenzentrierten Beratung, insbesondere im stationären Handel. Produktempfehlungen auf Basis persönlicher Daten bergen allerdings große Herausforderungen. Die Sammlung, Auswertung, Interpretation und Bereitstellung der Daten erfordern ein hohes Maß an Verantwortung im Bereich Datenschutz. Experte 3 äußerte sich zu diesem Thema folgendermaßen: „Ich glaube, da gilt es, so wenig Datenpunkte wie möglich in Anspruch zu nehmen, um das [= personalisierte Produktempfehlungen] zu erreichen ..., sodass es nicht creepy wird und nicht so quasi überwachungsstaatsmäßig wird, muss man 
da recht vorsichtig sein, was man alles für Daten in Anspruch nimmt, damit der Kanalübertritt gewährleistet werden kann“. Experte 4 bezeichnete eine transparente, glaubwürdige Datenstrategie als die zentrale Voraussetzung, um digital personalisierte Produktempfehlungen zu ermöglichen.

\subsubsection{Kundenbewertungen}

Die Bedeutung von Kundenbewertungen wurde in den Interviews mit Kunden wie auch Experten abgefragt. Eine hohe Relevanz zeigt sich in den Antworten einiger Experten auf die Frage, welche kaufrelevanten Informationen mit der digitalen Transformation verfügbar geworden sind. Experte 1 und 2 bezeichneten die Kundenwertungen sowie Fragen und Antworten von Kunden als eine der wichtigsten kaufrelevanten Informationen. Experte 3 bestätigte, dass FURNIT an eine Bedeutung von Kundenbewertungen glaube. So sei FURNIT einer der ersten Anbieter gewesen, die Kundenbewertungen einführten. Er präzisierte weiter: „Das hat definitiv einen Einfluss auf die Kaufentscheidung, auch wenn es eher vielleicht unterbewusst ist und nicht einmal so , ah, okay, und jetzt hat es ein Review, jetzt kaufe ich'. Sondern es ist eher nochmals so ein zusätzlicher Soft Factor, der dir hilft, deine kognitive Dissonanz zu nehmen“. Experte 2 ging noch einen Schritt weiter und erklärte: „Ich glaube, die größte Herausforderung liegt heute da drin, dass man Informationen, die online verfügbar sind, auch offline verfügbar macht. Ich denke da weniger an die ganzen Detailinformationen. ... Es geht vielmehr um die Frage, was eigentlich die beliebtesten Regale in unserem Onlineshop sind. Was sind die Topseller? Und genau so wichtig: Was sind denn eigentlich die Bewertungen. Was ist die Einschätzung unserer Kunden? ... Man könnte so elektronische Preistags verwenden, wo immer automatisiert die Sachen drauf sind. Oder man stellt irgendwie andere Medien zur Verfügung, wo der Kunde gleich ganz vertrauensfördernd die ganzen Kundenmeinungen, -bewertungen und auch Fragen und Antworten in der Offlinefiliale lesen kann“. Dass es in diese Richtung gehen könnte, verriet Experte 3: „Irgendwann werden unsere Produktschilder [und] unsere Preisschilder nicht mehr gedruckt sein. Sondern irgendwann wird das elektronisch sein. Und entweder werden sie dann schon ersichtlich sein und sonst haben wir irgendeinen NFC-Chip drin oder einen QR-Code, den man abscannen kann, und dann wird das Review dann spätestens auf dem Mobile Phone ersichtlich sein“.

Viele Kunden und Experten sind gegenüber Bewertungen von unbekannten Dritten skeptisch. Einige gaben an, sie würden die Bewertungen vor allem beachten, wenn sie negativ seien. Der Begleiter einer Interviewpartnerin bei FURNIT erklärte seine Bedenken folgendermaßen: „Ich habe kein großes Vertrauen in diese Kommentare, da man Kommentare auch selbst erstellen kann. Also jeder und alle können Kommentare erstellen. Ein Geschäft könnte auch sagen, dass sie einige Leute aussuchen, die eine gute Kritik schreiben sollen“. Interessanterweise bezeichneten insbesondere die Kundinnen, die sich über soziale Medien inspirieren lassen oder schon online Möbel gekauft hatten, Kundenbewertungen als relevant. Dies lässt die Vermutung zu, dass die Bedeutung bei der digital affinen Kundengruppe durchaus vorhanden ist. Überraschend kritisch äußerte sich der Experte für digitale Transformation, Experte 4: „Also da bin ich skeptisch. ... Man sieht es auch bei 
der Relevanz von Kuratoren oder Influencern, die füllen genau diese Lücke, weil man auch durch diese Massenbewertung einfach [beeinflussen kann]. Das ist hochgradig unseriös und die Schlussfolgerungen extrem schwierig, weil man dazu viel mehr wissen müsste, welche Person das gemacht hat. Also ich glaube, gerade im Möbelbereich, wo es um subjektive Einschätzungen geht in Bezug auf Stil und Ästhetik, [sind Kundenbewertungen] eher unwichtig, ja“. Er fügte aber an, dass er Prozessbewertungen von Kunden, also die Art des Austausches mit der Firma bzgl. Lieferungen, Reklamationen, Umgang mit Reklamationen, als hochgradig relevant einschätze.

\subsubsection{Nachhaltigkeit}

Nachhaltigkeit wird als Einflussfaktor wichtiger. Eine Kundin bei FURNIT erklärte: „Ich habe mich bei den Kissen bewusst entschieden, ... da sie aus natürlichen Fasern hergestellt wurden und nicht aus China stammen. ... Es hatte Kissen mit einem Siegel bezüglich Qualität, dass es eben fair produziert wurde. Die haben etwas mehr gekostet. Aber das konnte ich zahlen. Das war mir wichtig“. Diese Aussage zeigt, dass Kunden in gewissen Fällen bereit sind, mehr für nachhaltige Produkte zu zahlen. Fast alle Kunden gaben bei Möbel ITURE an, dass ihnen Nachhaltigkeit wichtig sei. Experte 3 erklärte die Relevanz von Nachhaltigkeit beim Kaufentscheid folgendermaßen: „Das ist sicher so etwas, das im Hintergrund schimmert und eben eigentlich so ein bisschen ein Halo-Effekt ist und dir einfach ein gutes Gewissen oder so die Bestätigung gibt: ,Ja, es ist etwas, ich mache es richtig ', in dem Sinne: ,Es ist das richtige Produkt'. Ich glaube eben, wenn der Preis nicht stimmt oder das Produkt nicht verfügbar ist, dann schlägt das aber diese Faktoren. Aber ich glaube, je länger, [desto] mehr wird das auch verlangt und wird auch wichtiger. Man hat jetzt auch gerade einen Test am Laufen mit dem FSC-Holz, zusammen mit anderen Ländern, aber es gibt da jetzt noch keine Insights, was das jetzt genau für eine Auswirkung hat auf den Kaufprozess". Experte 1 erwähnte Nachhaltigkeit im Zusammenhang mit Geschichten um das Produkt, um Kunden zu inspirieren. Auch Experte 4 wies auf das höhere $\mathrm{Ma} 3$ an Sensibilisierung zum Thema Nachhaltigkeit hin. Bezüglich des Kaufentscheidungsprozesses lässt sich zusammenfassen, dass Nachhaltigkeit ein Faktor ist, der für den Entscheid für ein Möbelstück nicht entscheidend ist, aber diesen begünstigen und die Zufriedenheit in der Nachkaufphase positiv beeinflussen kann.

\subsubsection{Konfiguration/Individualisierbarkeit}

Individualisierung ist ein wichtiger Trend in der Möbelbranche und es wird mit einer ansteigenden Nachfrage nach individualisierten Möbelstücken gerechnet (PwC 2017, S. 28). Auch der Branchenvertreter Experte 5 erwähnte im Zusammenhang mit der digitalen Transformation die besseren Möglichkeiten zur Konfiguration von Möbelstücken. Mit einer kundenspezifischen Konfiguration von Möbeln kann die Sicherheit, das passende Möbelstück zu kaufen, maßgeblich erhöht werden. Anders als bei Standardmodellen kann ein Teil der kaufrelevanten Informationen (Produktmerkmale) so verändert werden, dass sie besser auf die eigenen Bedürfnisse zugeschnitten sind. Trotzdem gilt es zu hinterfragen, wer unter Anbetracht der vorhandenen riesigen Auswahl sowie der Informationsflut die 
Konfiguration von Möbel vornehmen sollte. Kunden sind in vieler Hinsicht schon jetzt überfordert. Im Rahmen der Studie wurden einige Tests sowie anekdotische Beobachtungen durchgeführt. Die Erfahrung bei der Anwendung wurde dabei als nicht zufriedenstellend eingestuft. So waren die Funktionen nicht selbsterklärend und es gab zu wenig Hilfemöglichkeiten, um die Bedienung zu erlernen. Während der anekdotischen Beobachtungen in der Filiale von FURNIT fiel auf, dass auch einfachste Konfiguratoren nur gemeinsam mit Kundenberatenden genutzt wurden. Jedoch sind in diesem Zusammenhang weitere Untersuchungen nötig, um den Nutzen von Konfigurationstools praxisgerecht darzustellen.

\subsubsection{Kanäle und Kundenlenkung}

Bisher wurden Inspiration, Herausforderungen, sowie kaufrelevante Informationen beim Möbelkauf beleuchtet. In diesem Kapitel wird der Fokus auf die Nutzung der Kanäle beim Kaufentscheidungsprozess gelegt. Die Struktur des Kapitels folgt dem Ablauf der Customer Journey. Nach den Ausführungen zu den Erfolgsfaktoren für die Bereitstellung der Information auf digitalen Kanälen (Information online), wird die Gestaltung des Übergangs beschrieben. Schließlich werden die Erfolgsfaktoren für die Bereitstellung von Information im stationären Handel analysiert. Im Kapitel zum stationären Handel sind zudem die Ergebnisse der Analyse von Leading Practices zusammengefasst.

\subsubsection{Information online}

Neun der 13 interviewten Kunden hatte in der letzten Zeit den Onlineshop von Möbel ITURE oder FURNIT besucht. Sechs Kunden hatten bereits einmal online Möbel bestellt. Bezüglich der Angabe zu Onlinekäufen ist jedoch zu beachten, dass nicht genau spezifiziert wurde, um was für Möbel es sich handelt. So erklärte ein Kunde: „Ich kaufe mir höchstens eine Lampe auf Websites“. Somit lässt sich nicht abschließend eruieren, ob diese Kunden auch Möbelstücke mit hohem Involvement beim Kaufentscheidungsprozess online kaufen würden. Zudem ist nicht klar, ob die Kunden, die online bestellten, nicht davor bereits im stationären Handel Informationen zusammengetragen hatten. So meinte Experte 3 auch: „Wenn es ein Einkauf ist, wo man höher involviert ist, wird man wahrscheinlich selten nur einen Touchpoint angehen“. Experte 4 sagte dazu: „Speziell bei Möbeln ist natürlich, dass nebst dem Preis die ästhetische Ebene eine ganz hohe Relevanz hat. Und auf der bis heute noch beschränkten Abbildungsmöglichkeiten, nämlich auf einem zweidimensionalen Screen, kann diese ästhetische und haptische Ebene - wie fühlt sich ein Material an, wie wirkt es im Kontext des eigenen Zuhauses - nur sehr unbefriedigend visualisiert oder virtualisiert werden“.

Alle Experten erwähnten, dass bezüglich des Wegs zum gewünschten Objekt Optimierungspotenzial bestehe. Dabei scheint einerseits der Einstieg bzw. der Zugang zu den Produkten beispielsweise in Form von kundenzentrierten anstatt produktzentrieren Filtern und Suchfunktionen hilfreich. Andererseits wiesen mehrere Experten auf den Nutzen von Kuration hin. Dabei wird nicht anhand von Filtern oder Suchfunktionen die Auswahl 
eingeschränkt, sondern anhand einer Expertenmeinung Produkte empfohlen. Experte 1 erläuterte den Nutzen des Kuratierens folgendermaßen: „Es hat dann wie mehr Wert, wenn man sagt: ,Hey, dieser Tisch, der ist auf dich zugeschnitten, weil da können deine Kids rumturnen". Also mehr so Sachen, die im Alltag dann eine Relevanz haben“". Also mehr so Sachen, die im Alltag dann eine Relevanz haben“. Zudem wies Experte 1 darauf hin, dass dank der Einteilung von Produkten nach Bedürfnissen oder Lebensstilen auch die Produktinformation treffender auf die entsprechende Kundengruppe zugeschnitten werden könne. Dass mit einer Reduktion der Auswahl und somit Reduktion der Komplexität durch Expertenmeinungen ein gefragter Mehrwert geschaffen werden kann, zeigt ein Beispiel, das Experte 4 erwähnte: „Was ich noch interessant finde, ist die Plattform goodcheapandfast.com. Das ist ein Typ, der selektiert aus dem Amazon-Angebot in verschiedenen Kategorien Produkte. Also der reduziert sozusagen die Komplexität, bildet die Produkte auf seiner Website ab. Er wählt aus und wenn man es kaufen will, wird man auf Amazon geleitet. ... Die Leute kommen auf diesen Plattformen nicht mehr zurecht. Das ist zu abstrakt. Die Auswahl ist unkonsumierbar“. Als weitere Möglichkeit zur Reduktion der Komplexität scheinen wiederum automatisierte Produktempfehlungen zu sein. Auch Live-Chats oder Videoberatungen mit Kundenberatenden haben Zukunftspotenzial. Dies einerseits, weil im Rahmen der angestellten Beobachtungen starke Schwankungen beim Kundenaufkommen im stationären Handel festgestellt werden konnten und dadurch wohl vereinzelt Kapazitäten für zusätzliche Onlineberatungen bestehen könnten. Andererseits wäre Unterstützung bei der Suche, z. B. in Form der Beantwortung von Fragen oder auch beispielsweise bei der Anwendung eines Konfigurators, im richtigen Moment voraussichtlich hilfreich. Es gilt jedoch zu beachten, dass die allermeisten Kunden der Idee der Videoberatung oder Beratung über Chat sehr kritisch gegenüberstanden.

\subsubsection{Gestaltung Kanal-Übergang}

Kunden sollen in die Filiale gelenkt werden. So führte Experte 3 aus: „Aber natürlich, es ist Fakt, dass, wenn du im Store bist, die Conversion Rate um ein x-Faches höher als online ist. ... Und wie gesagt, der Kunde soll dann entscheiden, wie er die Customer Journey gestaltet. Wir probieren einfach möglichst, die Connection zwischen den verschiedenen Kanälen zu machen mit unseren Systemen, so gut, wie das halt eben möglich ist“". Diese Relevanz unterstrich auch Experte 1: „Das trägt dann viel stärker zur Loyalität bei. Ein Live-Kontakt ist immer intensiver als jeder Onlinekontakt, weil einfach so viel andere Sachen noch mitspielen. Man kann am POS mit so viel mehr arbeiten. Man hat persönlichen Kontakt zu den Leuten. Man kann einen Duft schaffen, der viel stärker oder der viel tiefer ins Hirn geht. Was eben die Leute viel stärker bindet ...". Bezeichnend dafür ist eine Aussage einer Kundin bei FURNIT. Sie sagte zum Grund ihres Filialbesuchs: „Wir hatten für heute nichts geplant und da sagte ich: ,Lass uns mal zu FURNIT gehen“". Es ist folglich nicht so, dass die Kunden lediglich wegen fehlenden Informationen zu einem Filialbesuch gezwungen werden, wie dies etwas überspitzt in der Costumer Journey formuliert war.

Ein gut gestalteter Kanalwechsel zum stationären Handel kann den Kaufabschluss begünstigen. Die meisten Kunden mussten sich für den Filialbesuch nicht vorgängig infor- 
mieren. Falls doch, wurden nötige Angaben wie die Verfügbarkeit des Wunschobjekts, Öffnungszeiten oder Adresse gefunden. Eine höhere Bedeutung hat jedoch, die Kunden zu einem Besuch des stationären Handels zu motivieren. So relativierte Experte 4 in diesem Zusammenhang das Ziel, den Abschluss bereits online herbeiführen zu wollen, folgendermaßen: „Naja, es ist doch schön, wenn es funktioniert, wie gesagt. Aber was mir nichts bringt, ist, wenn es die meisten wieder zurückschicken, weil es nicht den Vorstellungen entspricht. Also ich glaube, in einem ersten Schritt wäre es cooler, die Plattformen so zu nutzen, dass man sie als Vehikel in Richtung Möbelgeschäft nutzt, um dann entweder dort die Kaufentscheidung zu treffen oder aber dann, nachdem man es gesehen hat, zu Hause online, die finale Entscheidung noch mal entlang der Auswahlmöglichkeiten zu Ende führt. Das ist doch das, was gerade im Hochpreissegment funktioniert heute“. Der Übergang soll somit möglichst motivierend gestaltet werden, um online noch unentschlossenen Kunden das Fortführen des Kaufentscheidungsprozesses zu ermöglichen sowie zusätzlich die Chance zu nutzen, die Kundenbindung mit einem intensiven Kundenerlebnis zu erhöhen.

Die Motivation zum Besuch des stationären Handels sollte von positiven Emotionen begleitet werden. So erklärte Experte 3: „Ein Storebesuch bei uns soll auch immer ein Erlebnis sein. Also es soll auch immer so ein Überraschungsfaktor mitspielen. ... Eben, es soll ein Erlebnis sein ..., dass du auch wirklich eintauchst in verschiedene Welten und auch inspiriert wirst von unseren Raumsets und von anderen Ideen rund ums Zuhause. Und natürlich auch der Bauch, der mitkommt, der vielleicht auch einmal gefüllt werden muss zwischendurch, oder vorher oder nachher, in unserem Bistro oder im Restaurant. ... So probieren wir auch immer, die Überraschungs- oder Erlebniskomponente mitzugeben, wenn wir sagen: ,Okay, gehe doch in unseren Store schauen und mach dir einen schönen Tag im Store “.. Experte 1 wies in diesem Zusammenhang darauf hin, dass es viele Wege gäbe, jemanden für etwas zu motivieren und dies nicht unbedingt jedes Mal ein Preisnachlass, sondern Neugier oder Mitteilungsbedürftigkeit sein kann. Ebenfalls einen konkreten Vorschlag, der jedoch eher mit dem Preis zu tun hat, äußerte Experte 2: ,... ihnen vielleicht [online] gleich schon einen Bonus liefern: ,Jetzt guckst du dir diesen Artikel an und wir geben dir einen einmaligen Rabattcode und wenn du innerhalb der nächsten 5 oder 3 Tage in der Filiale den Rabattcode zeigst, dann bekommst du 5 \% extra““. Er erläuterte darauffolgend, wieso dies aus seiner Sicht wichtig ist: „Dann hätte man auch mal die Möglichkeit, das zu messen. Da scheitern ja auch viele daran. ... Diesen Impact für ROPO Research Online, Purchase Offline. ... Da muss man sich heute auch mal Gedanken machen, wie man das messbar machen kann". Um den Filialbesuch mit positiven Emotionen zu verknüpfen, kann den Kunden folglich ein Erlebnis (z. B. Überraschung, Essen, Kinderspielplatz, Events) oder einen Vorteil (Rabatte, Gewinnspiel) in Aussicht gestellt werden.

\subsubsection{Information stationär und Verknüpfung der Kanäle}

Ist der Übergang in den stationären Handel geschafft, sollten die Kunden nahtlos an die Onlinerecherche anschließen können und nicht wieder von vorne beginnen müssen. Wie im Ausschnitt der hypothetischen Customer Journey dargestellt, ist dieser nahtlose An- 
schluss aktuell kaum gewährleistet. Gleichzeitig wollen viele Kunden keine zusätzlichen Hilfsmittel oder Unterstützung. Eine Kundin bei Möbel ITURE meinte: „Ich dachte einfach, ich gehe von zuoberst nach unten durch. Diese Zeit nehme ich mir“. Auf die Frage, wie er auf seine vorgängig gesammelten Informationen in der Filiale zurückgreife, antwortete ein Kunde bei Möbel ITURE spöttisch: „Indem man sich daran erinnert“. Die 5 Kunden, die vorgängig online recherchiert hatten, gaben auf die Frage, wie gut sie in der Filiale an der Onlinerecherche anschließen können, 4-mal mit dem maximalen Wert als sehr gut an. Nur ein Kunde bei FURNIT beurteilte den Anschluss mit einer 3 eher mittelmäßig. Dieser Kunde befürchtete, dass das infrage kommende Möbel nicht passe und er dann von vorne beginnen müsse. Er fügte seiner Antwort aber an, dass er auch nicht wüsste, wie man die Situation verbessern könnte. Eine Kundin gab als Herausforderung beim Möbelkauf an, dass sie „Angst habe, [das Möbel] nicht zu finden“. Ihr Lösungsvorschlag wäre, das Personal zu fragen.

\section{Relevanz der Beratung}

Die Aussage der FURNIT-Kundin weist auf die Beratungskompetenz und den Informationsvorteil der Kundenberatenden als Erfolgsfaktor. Anders als die Kunden bestätigten nämlich mehrere Experten, dass der Anschluss an die Onlinerecherche wichtig sei und diesbezüglich Optimierungspotenzial bestehe. Die Frage, wie gut Kunden im stationären Handel auf die vorgängig recherchierten Informationen zugreifen (bzw. wieder anschließen) können, beurteilte Experte 2 mit 2 (eher ungenügend) und Experte 4 mit 1 (ungenügend) als sehr schlecht. Experte 4 führte aus: „Ich sehe überhaupt keine Schnittstelle. Vielleicht gibt es irgendwelche Computer, wo die Sachen da wären, aber der entscheidende Schritt ist ja, dass die Informationen dann zum Berater, zum Verkäufer gelangen. Und das habe ich bislang nie erlebt, dass dies wirklich gemacht wird“. Daraus lässt sich vermuten, dass es beim nahtlosen Anschluss an die Onlinerecherche vor allem um die weiterführende Beratung geht. Gleich mehrere Experten bemängelten, dass die Kundenberatenden vielfach kaum Informationen hätten, die nicht auch online abgerufen werden könnten. Experte 2 erklärte beispielsweise: „Das ist ja sehr oft leider dann doch nicht so, dass der Verkäufer einen beraten kann. Dass man heutzutage losgeht und viel mehr Informationen hat über das, was man kaufen will als die Verkäufer vor Ort“. Auch Experte 1 erwähnte, dass ihr ein Verkäufer nach der Onlinerecherche nicht viel zusätzliche Information liefern könne.

Insgesamt gaben die Kunden zur Relevanz der Beratung beim Möbelkauf mit einem Mittelwert von 4,4 sehr hohe Werte an. Bemerkenswert ist, dass 2 jüngere Kundinnen bei FURNIT, die wohl eher digital affin sind, mit 3 (weder wichtig noch unwichtig) als einzige einen eher tiefen Wert angegeben haben. Bei der geringen Anzahl befragter Kunden kann dies aber auch Zufall sein. Eine Kundin bei Möbel ITURE erzählte: „Vorher sah ich einen Tisch und sagte, in einem nächsten Schritt würde ich mich beraten lassen, damit mir die Möglichkeiten aufgezeigt werden und ich finde ein persönliches Gespräch manchmal bequemer als alles selbst online zu überlegen und zu machen“. Da diese Kundin vorgängig online recherchiert hatte, gälte es, laut Experte 4, genau hier anzusetzen: „Und die Kür 
wäre dann natürlich, die Themen, die man online recherchiert hat, oder sozusagen schon die Orientierung, die man hat, in den stationären Laden zu transferieren, dass man dort nicht wieder bei null anfängt. Das ist ja das, um was es heute geht, und was auch noch völliges Neuland ist". Um dies nutzenstiftend und damit kundenzentriert bewerkstelligen zu können, müssten nicht nur ein Merkzettel mit einzelnen Produkten, sondern auch der Weg, wie die Kunden zu den entsprechenden Produkten gekommen sind, von den Kundenberatenden nachvollzogen werden. Experte 4 erklärte dies folgendermaßen: ,Wenn ich nur die Empfehlungen sehe, habe ich keine Ahnung, was das für [ein Mensch] ist. Aber wenn ich weiß, zu was [sie] vorher ,ja “ und ,nein ' gesagt hat, dann kann ich mir sozusagen eine plastischere Vorstellung machen. Und diesen Weg mitnehmen können, damit jemand vor Ort, eine Person, ja, das als Grundlage für die weitere Unterstützung nehmen kann, das ist glaube ich der Clou“. Auch Experte 3 war sich diesem Optimierungspotenzial bewusst: „Nein, also eben, das ist sicher so, die digitale Kompetenz der Leute auf der Fläche, das ist sicher noch eine Herausforderung, die es zu lösen gilt. Also dass man ... ja, schlussendlich hängt alles an Daten. Und dass man die Daten, die relevant sind für die Mitarbeiter auf der Fläche, dass man die zum richtigen Zeitpunkt zur Verfügung stellen kann. Ob das jetzt mit einer App sei oder wie auch immer das dann aussehen wird, da arbeiten wir daran. Also da sind wir sicher noch nicht dort, wo wir sein wollen“. Es ist somit anzunehmen, dass mit der voraussichtlich zukünftig stattfindenden konsequenteren Datensammlung sowie der steigenden Fähigkeit, diese Daten zu interpretieren und zu nutzen, auch im stationären Handel vermehrt Orientierung in Form von bedürfnisspezifischer Beratung und Empfehlungen geboten wird. Diverse Trends der digitalen Transformation werden diesbezügliche neue Möglichkeiten mit sich bringen. Dies sind wohl beispielsweise das Internet of Things zur Datensammlung, Big-Data-Modelle und künstliche Intelligenz für die Auswertung und Analyse der Daten. Beratung bleibt also wichtig, sollte jedoch stärker die individuelle Customer Journey berücksichtigen.

\section{Verknüpfung der Kanäle und Beispiele Leading Practice}

Für das Bestreben, anhand von Nutzerdaten mehr Orientierung im Angebotsdschungel und der Informationsflut zu schaffen, müssen Kanäle miteinander verknüpft werden. Einerseits könnten Kunden direkt in der Filiale über Apps auf ihren Smartphones kundenspezifische Empfehlungen zur Verfügung gestellt werden. Dies z. B. abhängig davon, was sich die Kunden vorher online angeschaut oder in welchem Bereich der Filiale sie sich aufgehalten haben. Ähnlich könnte die Beratung funktionieren. In diesem Fall müssten die Nutzerdaten jedoch den Kundenberatenden zur Verfügung gestellt und in die Ausbildung des Beratungspersonals investiert werden.

Um was es bei der Verknüpfung der Kanäle geht, erläuterte Experte 2: „Da steckt eine ganze Menge im Detail. Man muss hinbekommen, dass Omnichannel nahtlos funktioniert, um verlässliche Informationen zu bekommen. Ist dieser Artikel im Lager verfügbar und in welcher Größe ist er verfügbar? Das gibt es ja oft schon, dass ich mir den [Artikel] reservieren kann. Dass ich nicht Gefahr laufe, dass er dann weg ist. Da sehe ich, dass man bei Möbel ITURE auch gleich einen Beratungstermin vor Ort buchen kann. Also da gibt es ganz 
viele Sachen, die ineinander spielen müssen“. Zum besseren Verständnis der Verknüpfung von Kanälen soll ein konkretes Beispiel im Zusammenhang mit den Kundenbewertungen skizziert werden. Um an mehr Kundenbewertungen zu gelangen, könnten beispielsweise auf den Kaufquittungen der Link zur Beurteilung der entsprechenden Produkte online enthalten sein. In der Handlungsaufforderung, Bewertungen online abzugeben, könnte wiederum eine Belohnung beispielsweise in Form eines Gutscheins für einen Kaffee oder ein Mittagessen in der Filiale enthalten sein. Damit könnte allenfalls erreicht werden, dass online mehr kaufrelevante Informationen zur Verfügung stehen. Gleichzeitig trägt man zur Kundenbindung bei, weil die Kunden motiviert werden, die Filiale wieder zu besuchen.

Es erscheint weiter sinnvoll, aus dem stationären Handel auf die wichtigsten Inspirationsquellen hinzuweisen und einen Anreiz zu schaffen, sich die entsprechenden Touchpoints anzusehen. Dies kann der Katalog, der eigene Onlineshop oder auch eine bei einer Kundengruppe beliebte soziale Plattform sein. Weiter sollten Hilfsmittel, die Kunden beim Bewältigen der größten Herausforderungen unterstützen, zur Verfügung gestellt werden. Im Zusammenhang mit dem Gewinnen von Sicherheit könnten dies Anwendungen sein, mit denen online zusammengestellte Merklisten transportiert und im stationären Handel wiederverwendet werden können (beispielsweise FURNIT Store-App). Umgekehrt sollte auch im stationären Handel auf Informationen, die online vorhanden sind, zugegriffen werden können. Sei dies mit dem eigenen Smart Phone über QR-Codes bei den entsprechenden Möbelstücken (wie gut solche Codes auch immer funktionieren) oder über digitale Preisschilder.

\section{Weniger ist mehr}

Im Zusammenhang mit der wachsenden Komplexität aufgrund der großen Auswahl und der Informationsflut wies Experte 1 auf folgenden Punkt hin: „Bei Kleidern hat man ja auch rausgefunden, dass wenn man weniger Sachen in den Läden ausstellt, tendenziell mehr gekauft wird“". Dies könnte vermutlich auch ein Hinweis für den Möbelhandel darstellen. Experte 2 sprach dann auch von einem „Etikettensalat“ im stationären Handel. Tatsächlich stellte sich anlässlich der Beobachtungen sowie den Interviews die Frage, wer da den Überblick behält und welche Informationen überhaupt beachtet werden. So hing bei FURNIT beispielsweise eine vom Betrag her nicht passende Anzeige zum Lieferservice neben dem Preisschild eines Sofas (bis CHF 1500 würde der Lieferservice nur CHF 69 kosten). Da dies anscheinend niemandem aufgefallen war, lässt sich kritisch hinterfragen, ob nicht Informationen weggelassen werden könnten.

\subsection{Diskussion}

In der vorliegenden Studie wurden Erfolgsfaktoren für die kundenzentrierte Bereitstellung kaufrelevanter Informationen im Schweizer Möbelhandel analysiert. Basierend auf den empirischen Erhebungen und im Abgleich mit der Literatur werden nachfolgend die Ergebnisse diskutiert und Handlungsempfehlung abgeleitet. 


\subsubsection{Inspiration}

Die hohe Relevanz der Inspirationsphase wurde vor den empirischen Untersuchungen unterschätzt. Die Inspirationsquellen sind dabei sehr vielschichtig. Unterschiedliche Kundengruppen nutzen unterschiedliche Kanäle oder Kombinationen davon; die neuen Möglichkeiten schaffen hier neue Verhaltensmuster. Experte 1 meinte dazu zugespitzt formuliert: „Heute kann man eigentlich sagen, dass so die Crowd oder Communities zu einer Art Gott wurden, weil man sie vorher konsultiert, was man machen soll und nachher ein Foto postet, um sich wie ein Verdikt abzuholen. Also das ist ja eigentlich etwas, das dann darüber entscheidet, ob ich happy bin mit einem Produkt oder nicht“. Unabhängig vom Kanal sollte eruiert werden, welche Kundengruppen sich in welchem Umfeld bewegen. Zusätzlich ist es relevant, die Aktivität der Kundengruppe im entsprechenden Kanal sowie die Gründe dafür zu kennen. Dazu gehört, gerade in sozialen Medien, welche Inhalte auf der Plattform geteilt werden oder wie hoch die Aufmerksamkeitsspanne der Kundengruppe im entsprechenden Netzwerk ist. Abgeleitet davon scheint es ein Erfolgsfaktor darzustellen, die Produkte beispielsweise mit der Entstehungsgeschichte oder Geschichten von Menschen, die sie nutzen, in einen emotionalen Kontext zu setzen. Storytelling ist eine gute Möglichkeit, Kunden zu unterhalten, emotional abzuholen und damit Bedürfnisse zu wecken. Die folgende Einschätzung von Experte 3 von FURNIT zu Storytelling zeigt jedoch die Grenzen dieser Art Inspiration: „Sicher schön und [es ist] auch eine schöne Bereicherung. Kann auch sein, dass man so halt über eine Story irgendwie zu einem Produkt kommt. Aber ich glaube nicht, dass das dann [für] Hard Selling quasi Treiber sind. Ich glaube, da zählen dann wirklich Hard Facts, die einen größeren Einfluss haben“. Hier gilt es, eine bessere empirische Grundlage zu schaffen, um die Bedeutung von Storytelling für die Inspiration und den Möbelkauf besser einschätzen zu können. Es wird aber auf jeden Fall deutlich, dass klassische Produktbeschreibungen nur eine Variante darstellen und Kunden ihre Inspirationen stärker danach ausrichten, welcher sozialen Gruppe sie sich zugehörig fühlen und welche Verhaltensmuster dabei typisch sind.

\subsubsection{Herausforderungen beim Möbelkauf}

Im Laufe des Kaufentscheidungsprozesses von Möbeln versuchen die meisten Kunden, ausreichend Sicherheit für den Kaufentscheid zu gewinnen. Ein exemplarischer Ablauf des Kaufentscheidungsprozesses und damit verbunden typischen Herausforderungen ist in Abb. 16.3 dargestellt. Schon früh im Kaufentscheidungsprozess versuchen die Kunden abzuschätzen, ob das Möbelstück in die Bestimmungsumgebung passt [1]. Danach wird die Unsicherheit bezüglich diversen Produktmerkmalen, wie beispielsweise Preis, Qualität, Haptik oder Komfort reduziert [2]; in vielen Fällen wird dafür der stationäre Handel besucht. Beim Versuch, Sicherheit zu gewinnen, werden die Meinungen von Dritten eingeholt, insbesondere von Mitentscheidenden [3]. Im gesamten Prozess steht eine unüberschaubare Auswahl an Möbeln zur Verfügung. Diese Angebotsvielfalt sowie die Informationsflut gilt es 


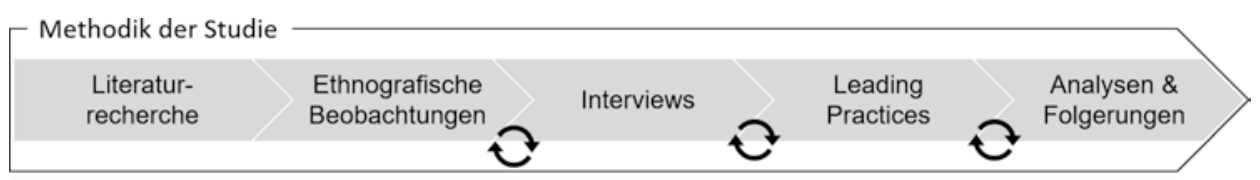

Forschungsfrage: Was sind die Erfolgsfaktoren für die kundenzentrierte

Bereitstellung kaufrelevanter Informationen im Schweizer Möbelhandel?

$\begin{array}{lccc}\text { Customer } & \text { Informationen } & \text { Gestaltung } & \text { Informationen } \\ \text { Journey } & \text { online } & \text { Übergang } & \text { stationär }\end{array}$

Abb. 16.3 Sicherheit bezüglich Kaufentscheidung entlang des Kaufentscheidungsprozesses. (Quelle: eigene Darstellung)

zu bewältigen und relevante Informationen herauszufiltern [4]. Schließlich können Informationen zu Möglichkeiten bezüglich Transport und Aufbau als Hygienefaktoren bezeichnet werden, die zusätzlich Sicherheit für den Kaufentscheid vermitteln [5]. Erst nachdem diese Herausforderungen bewältigt sind, kommt ggf. ein Kaufabschluss zustande.

Anhand der typischen Herausforderungen aus Sicht der Kunden lässt sich das kombinierte Modell des Kaufentscheidungsprozesses wiedererkennen. Das bereits zitierte Ehepaar bei Möbel ITURE sagte: „Möbel kaufen wir nicht jeden Tag und nehmen uns Zeit. Wir sind jetzt schon zum X-ten Mal unterwegs ...". Zu entscheiden, fällt offensichtlich schwer. Dies hat mit den relativ hohen Ausgaben, der guten Sichtbarkeit der Möbel bei sich zu Hause sowie der unüberschaubaren Auswahl zu tun. Weiter haben Kunden im Vergleich zu Alltagsgegenständen wenig Einkaufserfahrung. In Bezug auf die Customer Journey lässt sich festhalten, dass Kunden online aktuell relevante Informationen fehlen. Die Kunden erwähnten immer wieder, wie wichtig deshalb der stationäre Handel für sie sei. Es stellt sich daher die Frage, welche zusätzlichen Informationen online verfügbar gemacht werden sollten und wofür sich der stationäre Handel in Anbetracht der zur Verfügung stehenden Technologien weiterhin besser eignet.

Die Erreichbarkeit einer Filiale und Herausforderungen bezüglich der eigenen Mobilität führen dazu, dass ein Teil der Vorauswahl nicht infrage kommt. So verzichten Kunden darauf, den Weg zu einem Anbieter auf sich zu nehmen, wenn dieser als zu umständlich wahrgenommen wird. Möbelhändler wie FURNIT versuchen deshalb, mit dem stationären Handel näher an die Stadtzentren zu kommen. Die Herausforderung bezüglich des Transportes von Möbelstücken nach Hause sowie der Aufbau in den eigenen 4 Wänden steht am Schluss des Entscheidungsprozesses. Hier gilt es entsprechend attraktive Liefer-, Aufbauund Entsorgungsservices oder auch eine Lieferwagenausleihe zur Verfügung zu stellen, damit dieser Punkt den Kaufabschluss nicht behindert.

\subsubsection{Kaufrelevante Informationen}

Nach der Inspiration und der dazugehörigen Informationssammlung gilt es, den weiteren Prozess bestmöglich vorzubereiten. Kunden sollten angeregt werden, mit der nächsten Phase des Kaufentscheidungsprozesses weiterzufahren. Vor allem wenn reine Verkaufs- 
plattformen für die Präsentation der eigenen Möbel genutzt werden, besteht die Herausforderung, das Kundenerlebnis positiv zu prägen und die Mehrwerte der eigenen Marke zu transportieren. Experte 4 erklärte dazu: „Auf einer Plattform, nehmen wir Amazon, da wird ein Apfel genau so beschrieben, wie ein Regal. ... die Kategorien, in denen ein Produkt präsentiert wird, gelten für alle ...Sortimente, alle Produktkategorien. Und diese Abstraktion führt zu einer absolut eindimensionalen Abbildung von Produkten“. Kaufrelevante Informationen sollten phasenbezogen, aber kanalübergreifend angeboten werden. Der Ansatz, alles gleichzeitig zu präsentieren, wird den Ansprüchen von Kunden wenig gerecht. Inspirierende, positive Geschichten rund um Produkte, wie z.B. eine nachhaltige Herstellung, spielen vor allem zu Beginn des Kaufentscheidungsprozesses eine Rolle, schwingen in den Folgephasen mit und können am Schluss beim Entscheid das Zünglein an der Waage sein. Der Preis, die Verfügbarkeit und weitere Produktmerkmale sind bei der Informationssuche relevant. Informationen zur Garantie, einem Rückgaberecht, Umtauschangeboten, Wiederverkaufsmöglichkeiten und entsprechende Prozessbewertungen von Kunden reduzieren das Risiko bei einem vermeintlichen Fehlentscheid und können dadurch gegen Ende des Kaufentscheidungsprozesses die Wahrscheinlichkeit für einen positiven Entscheid erhöhen. Liefer- und Aufbauservices tragen, falls gewünscht oder nötig, zu einer Erleichterung unmittelbar nach dem Entscheid bei. Auch dies kann die Wahrscheinlichkeit eines Kaufabschlusses erhöhen bzw. Zweifel reduzieren. Dagegen wäre beispielsweise zu prüfen, ob sortimentsübergreifende Servicebedingungen oder auch Garantieleistungen in Onlineshops und insbesondere auch stationär tatsächlich, wie heute oft der Fall, direkt bei den Produkten angezeigt werden sollen, da sie keine eigentlichen Produktmerkmale darstellen. Allenfalls könnte die Frage nach solchen Bedingungen und Leistungen auch im Rahmen eines Check-ins zu Beginn beim Eintritt in die Filiale oder Besuch des Onlineshops geklärt und damit früh eine diesbezügliche Grundsicherheit erreicht werden. Dies würde jedoch eine einfache Angebotsgestaltung dieser Leistungen voraussetzen. Zudem wäre die tatsächliche Wirkung einer solchen Anpassung zu prüfen.

\subsubsection{Produktempfehlungen}

Ein bedürfnisorientierter Einstieg in den Kaufentscheidungsprozess über Filter- und Suchmöglichkeiten kommt eine besondere Bedeutung zu. Diese sollten kanalübergreifend möglich sein, jedoch aufgrund der Auswahl und damit verbundenen Menge an Informationen vor allem online bereitgestellt werden. Gleiches gilt für automatisierte oder auch von Experten ausgearbeitete, kundenspezifische Produktempfehlungen. Gemäß den Experten besteht hier großes Optimierungspotenzial und damit auch die Chance, damit einen Wettbewerbsvorteil zu erarbeiten. Ziel sollte es sein, dass Anbietende die Einrichtungsstile, die Vorlieben, das bisherige Einkaufsverhalten sowie die aktuelle Lebenssituation der Kunden kennen. Damit ließen sich zur Inspiration entlang des gesamten Kaufentscheidungsprozesses maßgeschneiderte Vorschläge oder Orientierungshilfen anbieten. Zudem sollten die entsprechenden Empfehlungen inkl. dem Weg dazu im richtigen Moment am richtigen Ort auch für die Beratung im stationären Handel verfügbar gemacht werden, was über eine Begleit-App für Smartphones möglich wäre. Dadurch würde sich der Prozess vereinfa- 
chen und voraussichtlich auch verkürzen lassen. Die bindende Wirkung zu Anbietenden liegt auf der Hand. Dafür bedarf es aber einer strukturierten Datensammlung, -auswertung und -nutzung sowie einer transparenten, glaubwürdigen Datenstrategie unter besonderer Berücksichtigung des Datenschutzes.

\subsubsection{Kundenbewertungen}

Kundenbewertungen werden in vielen Branchen genutzt und finden sich zunehmend auch in der Möbelbranche. Gleichzeitig wird deren Einfluss auf das Kaufverhalten unterschiedlich bewertet. Zwar sind sich viele Kunden der kaum einschätzbaren Seriosität der Bewertungen bewusst, jedoch können sie dennoch stark beeinflussen. Häufig findet eine positive Rückkopplung statt: Wird ein Produkt häufig positiv bewertet, vermittelt dies Sicherheit und erhöht die Wahrscheinlichkeit, der Bewertung zu trauen. Damit steigt potenziell die Chance, dass anhand der Beurteilung (unbekannter) Dritter ein Produkt online gekauft wird. Da ein Vergleich damit oft fehlt, wird zur Vermeidung von kognitiver Dissonanz das Produkt subjektiv als gut bewertet und damit die ursprüngliche Kundenbewertung bestätigt, ggf. mit einer eigenen Kundenbewertung. Es könnte allerdings sein, dass aufgrund der Sortimentsvielfalt sowie den im Vergleich zu Mode- oder Elektronikartikeln tieferen Absatzmengen oft keine aussagekräftige Anzahl Bewertungen zusammenkommt und andere, geeignetere Möbelstücke damit unberücksichtigt bleiben. Trotz dieser Vorbehalte kann von einer insgesamt positiven Wirkung von Kundenbewertungen für die Einschätzung der Produkteigenschaften und somit auf die Sicherheit im Entscheidungsprozess ausgegangen werden, v. a. wenn eine hohe Anzahl Bewertungen vorhanden ist. Dies könnte mit Anreizen für die Erfassung von Bewertungen oder einer Zusammenfassung von Bewertungen über ganze Linien oder Produktgruppen erreicht werden. Weiter könnten produktspezifischere Bewertungskategorien hilfreich sein, die sonst nur im stationären Handel beurteilt werden können. Konkret wären dies beispielsweise bei Sofas Bewertungskategorien für die Materialqualität oder zum Komfort. Sind einmal genug Bewertungen vorhanden, könnten diese auch im stationären Handel zur Unterstützung des Kaufentscheidungsprozesses zur Verfügung gestellt werden. Ein mögliches Trägermedium dafür wären elektronische Preisschilder. In diesem Zusammenhang muss beachtet werden, dass im stationären Handel wiederum andere Bewertungskategorien an Relevanz gewinnen. Können Materialqualität und Komfort dort selbst beurteilt werden, interessieren wohl eher Bewertungen zur Montage, Langlebigkeit oder Pflege des Sofas. Wichtig wäre deswegen, auch im stationären Handel mehr Möglichkeiten für Kundenbewertungen zu schaffen, z. B. über Feedbackmöglichkeiten an den elektronischen Preisschildern.

\subsubsection{Konfiguration/Individualisierbarkeit}

Bezüglich des Nutzens der selbstbedienten Individualisierbarkeit bzw. Konfigurierbarkeit von Möbeln ist ein gewisses Maß an Skepsis aufgrund der großen Auswahl angemessen. Zwar können die Produkte den eigenen Bedürfnissen entsprechend angepasst werden, jedoch erhöht dies die Komplexität im Kaufentscheidungsprozess zusätzlich. Anekdotische Beobachtungen legen außerdem nahe, dass der Prozess des Konfigurierens häufig abge- 
brochen wird, bevor ein Entscheid getroffen wurde und alternative Informationsquellen genutzt werden. Es könnte daher sein, dass die derzeitige Bereitstellung eines Konfigurierungstools eher zu einem negativen Erlebnis für Kunden wird. Es fehlen jedoch dazugehörige Daten, wie beispielsweise Nutzerzahlen oder Kundenbewertungen des Tools. Deshalb gilt es zu prüfen, ob solche Konfigurationen nicht im Sinne einer Beratung bzw. ähnlich wie bei Produktempfehlungen von Experten, in diesem Zusammenhang Kundenberatende, ausgeführt werden sollten. Um die Konfigurationsmöglichkeiten trotzdem auch online anzubieten, könnte eine kanalübergreifende Kundenberatung sinnvoll sein (Chat-, Videoberatung). Dabei gilt es aber, die sehr kritische Beurteilung dieser Ideen durch Kunden zu berücksichtigen. Beim momentanen Stand der Technik wird ein digitaler Möbelkonfigurator deshalb nicht als kundenrelevante Informationsquelle erachtet.

\subsubsection{Kanäle und Kundenlenkung}

„Wir wissen heute aufgrund verschiedener Untersuchungen, dass der Anteil des Umsatzes direkter Onlinekäufe bei ungefähr 7-8 \% liegt, aber dadurch noch ein relativ großes Entwicklungspotenzial nach oben hat. Im Vergleich zu elektronischen Artikeln, [da] ist der Anteil ungefähr bei 30-35 \%", erklärte Branchenvertreter Experte 5. Elektronische Artikel sind lediglich von der Preisklasse mit Möbel vergleichbar. Dennoch ist davon auszugehen, dass mit einer optimierten Bereitstellung von Informationen auch Möbel vermehrt direkt online bestellt werden. Vor allem, weil es potenziell Kundengruppen gibt, die sich durch eine detaillierte, hochauflösende, bewegte oder interaktive Darstellung der Produkte überzeugen ließe. Gerade weil Kunden Onlinebewertungen kritisch hinterfragen und sich zumindest teilweise der Subjektivität und der unterschiedlichen Aussagekraft bewusst sind, könnten wertvolle Rückschlüsse aus diesen Bewertungen abgeleitet werden, besonders wenn sie mit Fließtext-Kommentaren ergänzt sind. Mit einem bedürfnisorientierten $\mathrm{Zu}-$ gang bzw. Filter und Suchfunktionen oder Produktempfehlungen durch Experten oder Algorithmen kann zudem eine zielführende Informationssuche und Bewertung von Alternativen auf den digitalen Kanälen vereinfacht oder überhaupt ermöglicht werden.

In diesem Zusammenhang stellt sich jedoch die Frage, wie weit diese Entwicklung überhaupt von den Anbietenden forciert werden kann und soll. Besteht doch für Kunden wie auch für den Möbelhandel ein hoher Nutzen des stationären Handels. Voraussichtlich ist es so, dass trotz der erwähnten Optimierungen bei der Information online es für die meisten Kunden gute Gründe geben wird, den stationären Handel zu besuchen. Sei es, um sich live inspirieren zu lassen und etwas zu erleben, oder um sich doch bewusst mehrere infrage stehende Alternativen vor Ort ansehen zu wollen. Aufgrund dieser unterschiedlichen Vorgehensweisen beim Einkauf, die sich unter Umständen je nach Lebenssituation sogar beim einzelnen Individuum von Einkauf zu Einkauf wieder ändern können, gilt es von den Anbietenden alle Möglichkeiten abzudecken. Kunden sollen direkt online abschließen, aber auch vorinformiert oder völlig unvorbereitet mit einem konkreten Ziel oder nur zur Inspiration in die Filiale kommen können. Wichtig für die langfristige Kun- 
denbindung erscheint, dass sich der Anbietende diesen unterschiedlichen Voraussetzungen bewusst ist. Nur so können die Übergänge kundenzentriert gestaltet sowie die Kundenerlebnisse bleibend und bindend gestaltet werden. Ein kritischer Erfolgsfaktor wird dabei sein, der Vielfalt im Angebot und der Informationsflut zu begegnen und mit Empfehlungssystemen Vereinfachung sowie Orientierung zu schaffen.

Kunden möchten sich inspirieren lassen und einen Einkauf mit allen Sinnen erleben. Anbietende können mit der Schaffung von intensiven Einkaufserlebnisse, die tiefer gehen als der Onlineeinkauf, die Kundenbindung erhöhen. Indem Überraschungen, Erlebnisse und Preisvorteile in Aussicht gestellt werden, sind Anreize zu schaffen, damit der stationäre Handel besucht wird. Entsprechend bewusst sollten die Einkaufserlebnisse in den Filialen gestaltet werden. Eine Verknüpfung der Kanäle erscheint dabei sinnvoll, um die entscheidendsten kaufrelevanten Informationen aus anderen Kanälen verfügbar zu machen, grundsätzlich mehr über die Kundenbedürfnisse zu lernen sowie mit einer qualitativ hochstehenden, maßgeschneiderten Beratung einen Mehrwert zu schaffen. Dafür sind Kundenkarten und mobile Applikationen sinnvoll.

\subsubsection{Handlungsempfehlungen für die Praxis}

Nachfolgend werden, abgeleitet von den identifizierten Erfolgsfaktoren, konkrete Handlungsempfehlungen für die Praxis formuliert. Es gilt zu beachten, dass diverse Themen und Wirkungszusammenhänge nur oberflächlich untersucht werden konnten. Um die Effekte tatsächlicher Änderungen bei der Bereitstellung kaufrelevanter Information voraussagen zu können, sind daher weitere Untersuchungen notwendig. In Tab. 16.3 werden für alle Erfolgsfaktoren stichwortartige Maßnahmen und Vorschlägen zugeordnet.

Um diese Maßnahmen unter Berücksichtigung der Gesamtzusammenhänge anzugehen, wird Möbelhändlern empfohlen, ihre Organisationsstruktur kundenzentriert aufzustellen. Es bedarf dafür einer starken Marketingabteilung, die sich weniger nach Produktkategorien, sondern mehr an den Kundengruppen bzw. dem Kaufentscheidungsprozess ausrichtet. Das Channel-Management sollte dabei eine dirigierende Querschnittsfunktion übernehmen. Dies beinhaltet, dass Anforderungen an die weiteren Marketingfunktionen, wie beispielsweise das Produktmanagement oder die Marketingkommunikation, aber auch an die Weiterentwicklung der IT-Systeme, das Prozessmanagement, den Vertrieb bzw. Verkauf bis hin zur Personal- und Organisationsentwicklung, gestellt werden können. Dafür ist eine konsequent an den Kundenbedürfnissen ausgerichtete Grundhaltung im Unternehmen Voraussetzung. Verstehen sich alle Organisationsmitglieder abteilungs- und kanalübergreifend als Teil des Gesamtsystems, bestehen gute Aussichten, die Herausforderungen der digitalen Transformation erfolgreich zu meistern und die sich bietende Chancen zu nutzen. Startpunkt bildet das Verständnis der in dieser Studie grob erfassten Verhaltensmuster von Kunden beim Kaufprozess und deren Erklärungen. 
Tab. 16.3 Erfolgsfaktoren und dazugehörige Handlungsempfehlungen

\begin{tabular}{|c|c|}
\hline Erfolgsfaktor & Handlungsempfehlungen \\
\hline $\begin{array}{l}\text { Kenntnis der Herausforderungen } \\
\text { beim Möbelkauf }\end{array}$ & $\begin{array}{l}\text { - Kunden quantitativ und qualitativ (Interviews) befragen } \\
\text { - Feedback auf allen Kanälen ermöglichen }\end{array}$ \\
\hline $\begin{array}{l}\text { Emotionale Produktpräsentation } \\
\text { zur Inspiration (Storytelling)/ } \\
\text { Onboarding auf eigene Kanäle }\end{array}$ & $\begin{array}{l}\text { - Kanalnutzung je Kundengruppe erfassen } \\
\text { - Kanalspezifische Inhalte (Content) erstellen } \\
\text { - Mit Kundengruppen spezifisch interagieren } \\
\text { - Anreize für die Fortführung des Entscheidungsprozesses } \\
\text { auf eigenen Kanälen schaffen }\end{array}$ \\
\hline $\begin{array}{l}\text { Auf Kaufentscheidungsprozess } \\
\text { ausgerichtete Bereitstellung der } \\
\text { Information }\end{array}$ & $\begin{array}{l}\text { - Relevanz der kaufrelevanten Informationen laufend und } \\
\text { kanalspezifisch überprüfen } \\
\text { - Tests zur Eindämmung des „Etikettendschungels“ } \\
\text { durchführen (z. B. Trennung Information Service- und } \\
\text { Garantieleistungen von Produktspezifikationen) }\end{array}$ \\
\hline $\begin{array}{l}\text { Bedürfnisorientierte } \\
\text { Produktzugänge (Filter und Suche) }\end{array}$ & $\begin{array}{l}\text { - Erwartungshaltung bezüglich des Zugangs ermitteln } \\
\text { (z. B. Einrichtungsstil, Bedürfnisse, Lebenssituation) } \\
\text { - Dementsprechend Filter und Suchmöglichkeiten } \\
\text { überarbeiten (Taxonomie) }\end{array}$ \\
\hline $\begin{array}{l}\text { Produkteempfehlungen inkl. } \\
\text { transparenter, glaubwürdiger } \\
\text { Datenstrategie }\end{array}$ & $\begin{array}{l}\text { - Datenstrategie entwickeln } \\
\text { - Datenquellen identifizieren und verknüpfen } \\
\text { - Datensammlung/-auswertung planen und durchführen } \\
\text { - Daten aufbereiten und zur Verfügung stellen }\end{array}$ \\
\hline $\begin{array}{l}\text { Nahtlosen Anschluss an die } \\
\text { Onlinerecherche durch } \\
\text { Kundendaten für die Beratung }\end{array}$ & $\begin{array}{l}\text { - Beratungsprozesse und -hilfsmittel weiterentwickeln } \\
\text { - Mitarbeitende zu Kuratoren aus- und weiterbilden } \\
\text { - Daten auf Kundenberatende zugeschnitten aufbereiten } \\
\text { - Produktempfehlungen kundenspezifisch bereitstellen }\end{array}$ \\
\hline $\begin{array}{l}\text { Produktbewertungen online und } \\
\text { stationär }\end{array}$ & $\begin{array}{l}\text { - Aussagekräftige Bewertungskategorien definieren } \\
\text { (z. B. Komfort bei Sofas) } \\
\text { - Anreize zur Erfassung von Bewertungen schaffen } \\
\text { - Allenfalls Zusammenführung von Bewertungen über } \\
\text { Produktkategorien oder Linien prüfen } \\
\text { - Bewertungen im stationären Handel anzeigen } \\
\text { - Fokus auf kanalspezifisch relevante Bewertungskategorien }\end{array}$ \\
\hline $\begin{array}{l}\text { Bedienung der Konfiguration im } \\
\text { Sinne von Empfehlungen }\end{array}$ & $\begin{array}{l}\text { - Anwenderdaten der Konfiguratoren laufend auswerten } \\
\text { - Rückmeldung von Anwendern einholen } \\
\text { - Konfiguration rein bedient anbieten }\end{array}$ \\
\hline $\begin{array}{l}\text { Kanalübergreifenden } \\
\text { Beratungsdienstleistungen über } \\
\text { Video- oder Chatfunktionen }\end{array}$ & $\begin{array}{l}\text { - Rahmenbedingungen (z. B. Erreichbarkeit) festlegen } \\
\text { - Kundenberatende aus dem stationären Handel einbinden } \\
\text { - Akzeptanztests durchführen und auswerten }\end{array}$ \\
\hline
\end{tabular}

(Fortsetzung) 
Tab. 16.3 (Fortsetzung)

\begin{tabular}{l|l}
\hline Erfolgsfaktor & Handlungsempfehlungen \\
\hline Optimierte Bereitstellung von & - Produkte auch im Onlineshop mit Geschichten aufladen \\
Information online mithilfe & - Hochauflösende Bilder des Materials bereitstellen \\
immersiverer Produktpräsentation & - In Videosequenzen die Nutzung inszenieren und bewerten \\
\hline Aktive Lenkung in den stationären & - Gewünschtes Einkaufserlebnis bewusst konzipieren \\
Handel inkl. bewusste Gestaltung & - Anreizsysteme für den Filialbesuch schaffen \\
der Erlebnisse & (Überraschungseffekt, Preisvorteile, Events, \\
& Kinderbetreuung) \\
\hline Verknüpfung der Kanäle & - In Filiale auf Inspirationsquellen (z. B. soziale Medien) \\
& hinweisen und Anreize zur Nutzung schaffen \\
& - Glaubwürdige Datenstrategie transparent machen \\
& - Vorteile des Kuratierens vermitteln und Bereitschaft zur \\
& Preisgabe von Vorlieben, Einrichtungsstilen etc. fördern \\
& - Auf Applikationen und Kundenkarten hinweisen \\
& - Onlinerecherche für nichtregistrierte Kunden anonymisiert \\
& transportierbar machen \\
\hline
\end{tabular}

\subsection{Schlussfolgerungen und Ausblick}

Mit den empirischen Untersuchungen konnte festgestellt werden, dass es für Kunden beim Möbelkauf mit hohem Involvement herausfordernd ist, ausreichend Sicherheit für einen Entscheid zu gewinnen. Unsicherheit besteht in Bezug auf die Wirkung des Möbels in der Bestimmungsumgebung, der Produktmerkmale sowie der Meinung Dritter. Erschwert wird der Entscheid zusätzlich von einer schwer überschaubaren Auswahl und Informationsflut, was die Kunden überfordern, ermüden und Stress verursachen kann.

Mit der vorliegenden Untersuchung konnten diverse Erfolgsfaktoren für die kundenzentrierte Bereitstellung kaufrelevanter Information identifiziert werden. Verschiedene Wirkungszusammenhänge konnten aufgrund der breit angelegten Untersuchung sowie dem qualitativen Forschungsdesign lediglich oberflächlich beleuchtet werden. Beginnend bei den Herausforderungen beim Möbelkauf gälte es beispielsweise, den Einfluss der stark angewachsenen Auswahl und der Informationsflut genauer zu analysieren. Ist es tatsächlich so, dass Kunden mit der Angebots- und Informationsvielfalt überfordert sind und deshalb Stress und Ermüdungserscheinungen resultieren? Oder finden sich die Kunden doch besser zurecht, als dies von den Experten eingeschätzt wird?

Auch die Wirkung von Maßnahmen, mit denen dieser potenziellen Überlastung begegnet werden soll (z. B. Kuration), konnte im Rahmen der vorliegenden Forschung nicht überprüft werden. Ebenfalls offen bleibt, wie stark sich Kunden bei Entscheiden von Kundenbewertungen beeinflussen lassen und was eine Beeinflussung für Auswirkungen auf das Einkaufserlebnis hätte. Die in diesem Zusammenhang formulierte Hypothese, dass mit zusätzlichen (positiven) Kundenbewertungen die Onlineeinkäufe ansteigen könnten, bildet deshalb einen Ansatzpunkt für weitere Forschung. 
Da die Individualisierung als bedeutender Trend in der Möbelbranche angesehen wird (PwC 2017, S. 28), setzen Händler, wie beispielsweise Möbel ITURE, auf selbstbediente Konfiguratoren. Der Erfolg dieser Strategie ist jedoch noch unklar. Es ist aber durchaus denkbar, dass mit einer sehr einfachen, intuitiven Bedienbarkeit dieser Lösungen ein Kundenbedürfnis befriedigt wird. Um die Akzeptanz solcher Lösungen zu überprüfen und die Auswirkungen auf den Kaufentscheidungsprozess zu eruieren, ist ebenfalls weitere Forschung notwendig.

Auf einer höheren Ebene gilt es, die Auswirkungen der zunehmend besser digital abbildbaren Kunden zu beleuchten. In Anbetracht der bevorstehenden Entwicklung mit Big Data, künstlicher Intelligenz und dem Internet of Things nimmt die Nachverfolgbarkeit, aber auch die Prognostizierbarkeit der Handlungen, Interessen und Bedürfnisse ständig zu. Es sind damit kundenfreundliche Angebote und weitere Erleichterungen des Lebens theoretisch immer einfacher möglich: Zeichnet die Stehlampe auf, dass sie auf den Salontisch umgefallen und dadurch beide Möbelstücke beschädigt wurden, erhalten deren Besitzer innerhalb von Sekunden Produktempfehlungen, die das Wohnzimmer noch gemütlicher machen, als vor dem Missgeschick. Inspirieren lassen, Informationen suchen oder Alternativen vergleichen müssten Kunden immer weniger. Es ginge nur noch darum, die Lieferung auszulösen. Wie beeinflussbar Individuen in einer solchen Welt wären und ob dies eine wünschenswerte Zukunft bzw. allenfalls zum Teil auch schon Gegenwart ist, gilt es weiter zu untersuchen.

\section{Literatur}

Accenture. (o. J.). Product as a Service Circular Business Model - Accenture Outlook. https://www. accenture.com/us-en/insightoutlook-buy-own-thats-yesterdays-model. Zugegriffen am 02.06.2019.

Aemmer, D., Bigler, J., \& Birkhofer, M. (2019). Der Nutzen von Virtual Reality, AR / VR als Hilfsmittel beim Möbelkauf. Wiss. Praxisproject, Berner Fachhochschule BFH W.

Berghaus, S., Back, A., \& Kaltenrieder, B. (2017). Digital Maturity \& Transformation Report 2017. St. Gallen: Universität St. Gallen in Zusammenarbeit mit /Crosswalk/.

Die Schweizerische Post AG. (2019). Die Schweizerische Post AG, Geschäftsbericht 2018.

Felber, P. (2018). Die Migros-Tochter Interio rüstet sich mit einem neuen Ladenkonzept für die digitale Zukunft. az Aargauer Zeitung. https://www.aargauerzeitung.ch/wirtschaft/die-migrostochter-interio-ruestet-sich-mit-einem-neuen-ladenkonzept-fuer-die-digitalezukunft-132983314. Zugegriffen am 04.01.2019.

Fischbach, R., \& Wollenberg, K. (2014). Volkswirtschaftslehre I: Einführung und Grundlagen. Walter de Gruyter GmbH \& Co KG.

Frey, D., von Rosenstiel, L., \& Hoyos, C. G. (Hrsg.). (2005). Wirtschaftspsychologie (S. 364-369). Weinheim: Beltz (Beltz-Handbuch).

Friedrich, C., \& Diekmann, M. (2016). Auch Möbel geht online - Cross-Channel-Vision und digitale Adoption der Pfister AG. In G. Heinemann, H. M. Gehrckens \& U. J. Wolters (Hrsg.), Digitale Transformation oder digitale Disruption im Handel: Vom Point-of-Sale zum Point-of-Decision im Digital Commerce (S. 279-300). Wiesbaden: Springer Fachmedien. https://doi.org/1 0.1007/978-3-658-13504-1_14.

Fribus, T. (2004). Auswirkungen der Änderung von Rabattgesetzen u. ä. auf das Anbieter- und Nachfrageverhalten. diplom.de. 
Forrester. (o. J.). Forrester: Customer Journey. Customer Journey. https://www.forrester.com/Customer-Journey\#. Zugegriffen am 26.05.2019

Gelbrich, K., Wünschmann, S., \& Müller, S. (2018). Erfolgsfaktoren des Marketing. Vahlen.

Griese, K.-M., \& Bröring, S. (2011). Marketing-Grundlagen: eine fallstudienbasierte Einführung (1. Aufl.). Gabler (Lehrbuch): Wiesbaden.

Groissberger, T. (2014). Problemerkennung - 1. Phase des Kaufprozesses nach Kotler.

Groissberger, T. (2013). Webanalyse entlang des Kaufprozesses, Bachelorarbeit. FH Oberösterreich, Steyr.

Halbmayer, E., \& Salat, J. (2011). Strukturierung qualitativer Interviews, Institut für Kultur und Sozialanthropologie, Universität Wien. Qualitative Methoden der Kultur und Sozialanthropologie. http:// www.univie.ac.at/ksa/elearning/cp/qualitative/qualitative-31.html. Zugegriffen am 15.06.2018.

Heinemann, G., Gehrckens, H. M., Wolters, U. J., et al. (Hrsg.). (2016). Digitale Transformation oder digitale Disruption im Handel: vom Point-of-Sale zum Point-of-Decision im Digital Commerce. Wiesbaden: Springer Gabler.

Holland, H. (2018). Definition: Customer Experience Management. Gabler Wirtschaftslexikon Online. https://wirtschaftslexikon.gabler.de/definition/customer-experience-management-54478/version-277507. Zugegriffen am 14.06.2018.

ITU. (2018). New ITU statistics show more the half the world is now using the Internet. ITU News. https://news.itu.int/itustatistics-leaving-no-one-offline/. Zugegriffen am 20.05.2019.

Khan, H. (2018). Consumers are showrooming and webrooming your business. Shopify. https:// www.shopify.com/retail/119920451-consumers-are-showrooming-and-webrooming-your-business-heres-what-that-me-ans-and-what-you-can-do-about-it. Zugegriffen am 06.06.2019.

Kotler, P., Armstrong, G., Harris, L. C., et al. (2016). Grundlagen des Marketing (6., Ak. Aufl.). Hallbergmoos: Pearson Deutschland GmbH (wi - Wirtschaft).

Kirchgeorg, M. (2018). Definition: Involvement. Gabler Wirtschaftslexikon Online. https://wirtschaftslexikon.gabler.de/definition/involvement-39554. Zugegriffen am 16.06.2018.

Klaus, P. (2015). Measuring customer experience: how to develop and execute the most profitable customer experience strategies. New York: Palgrave Macmillan.

Kotler, P., Armstrong, G., Harris, L. C., et al. (2016). Grundlagen des Marketing (6., aktualisierte. Aufl.). Hallbergmoos: Pearson Deutschland GmbH (wi Wirtschaft).

Laesser, C. (2004). Das Kundenverhalten im Kaufprozess bei persönlichen Dienst-leistungen - das Beispiel Tourismus - Sammelhabilitation. St. Gallen: Universität St. Gallen, IDT-HSG, Institut für Öffentliche Dienstleistungen und Tourismus.

Leising, L. (2010). Modelle des Entscheidungsverhaltens von Konsumenten und ihre praktische Anwendung durch Test-Anbieter: Am Beispiel der Stiftung Warentest. diplom.de.

Lemon, K. N., \& Verhoef, P. C. (2016). Understanding customer experience throughout the customer journey. Journal of Marketing, 80, 69-96. https://doi.org/10.1509/jm.15.0420.

Levin, I. P., Johnson, R. D., \& Faraone, S. V. (1984). Information integration in price-quality tradeoffs: The effect of missing information. Memory \& Cognition, 12, 96-102.

Maier, G. W. (2018). Definition: kognitive Dissonanz. Gabler Wirtschaftslexikon. https://wirtschaftslexikon.gabler.de/definition/kognitive-dissonanz-37371/version-260807. Zugegriffen am 02.06.2019.

Martineau, P. (1958). Social glasses and spending behavior. Journal of Marketing, 23(2), 121-130.

Nelson, P. (1970). Information and consumer behavior. Journal of Political Economy, 78(2): 311-329.

Oberholzer, G., \& Leuthold, S. (2018). Customer Experience Monitor 2018. Stimmt.ch.

von Nitzsch, R. (2006). Entscheidungslehre. Mainz.

Patel, N. (2014). The psychology of instant gratification and how it will revolutionize your marketing Approach. Entrepreneur. https://www.entrepreneur.com/article/235088. Zugegriffen am 16.06.2018.

Peter, J. P., \& Olson, J. C. (2010). Consumer behavior \& marketing strategy (9. ed., internat. Aufl.). Boston: McGraw-Hill. 
Pepels, W. (2013). Käuferverhalten: Basiswissen für Kaufentscheidungen von Konsumenten und Organisationen; mit Aufgaben und Lösungen (2., neu bearb. und erw. Aufl.). Berlin: E. Schmidt.

Porter, M. E. (2008). The five competitive forces that shape strategy. Harvard Business Review, 25-40.

PostFinance AG. 2018. PostFinance AG Geschäftsbericht 2018.

PwC. (2017). Die deutsche Möbelbranche Struktur, Trends und Herausforderungen. (Branchenanalyse).

Raab, G., Unger, A., \& Unger, F. (2010). Die Theorie kognitiver Dissonanz. In G. Raab, A. Unger \& F. Unger (Hrsg.), Marktpsychologie: Grundlagen und Anwendung (S. 42-64). Wiesbaden: Gabler. https://doi.org/10.1007/978-3-8349-6314-7_4.

Rutschmann, M., \& Belz, C. (2014). Reales Marketing: Kunden zum Kauf führen; Kaufprozesse, Kaufhandlung, Erfolg. Stuttgart: Schäffer-Poeschel.

Schuchard-Ficher, C. (1979). Ein Ansatz, zur Messung von Nachkauf-Dissonanz. Duncker \& Humblot.

Schulz, M. (2014). Ethnografische Beobachtung. In Handbuch Kinder und Medien (S. 225-235). Wiesbaden. (Digitale Kultur und Kommunikation): Springer VS. https://doi.org/10.1007/978-3 -531-18997-0_17.

Smith, S., \& Milligan, A. (2015). On purpose: delivering a branded customer experience people love. London/Philadelphia: Kogan Page.

Statista. (o. J.). Topic: Mobile internet usage worldwide. www.statista.com. https://www.statista. com/topics/779/mobile-internet/. Zugegriffen am 20.05.2019.

Swisscom AG. 2019. Swisscom Geschäftsbericht 2018.

Traylor, M. B. (1983). Ego involvement and brand commitment: Not necessarily the same. Journal of Consumer Marketing, 1(2), 75-79.

Trommsdorff, V., \& Teichert, T. (2011). Konsumentenverhalten (8., vollst. überarb. u. erw. Aufl.). Stuttgart: Verlag W. Kohlhammer (Kohlhammer Edition Marketing).

Verband Schweizer Möbelhandel und -industrie. (2017). Die aktuelle Situation in der Schweizer Möbelbranche - und ein Ausblick. Verband möbelschweiz. https://www.xn--mbelschweiz-rfb. ch/de/liste_229490-603664/die-aktuelle-situation-in-der-schweizer-moebelbranche---und-ein-ausblick.html. Zugegriffen am 16.06.2018.

Verband Schweizer Möbelhandel und -industrie. (2019). Die aktuelle Situation in der Schweizer Möbelbranche-und ein Ausblick. https://www.xn--mbelschweiz-rfb.ch/de/liste_229490-782078/ die-aktuelle-situation-in-der-schweizer-moebelbranche--und-ein-ausblick.html. Zugegriffen am 05.06.2019.

Verband der deutschen Möbelindustrie. (2018). VDM-Studie zum „Wohnen in Deutsch-land“ Preis beim Möbelkauf weniger wichtig. https://www.moebelkultur.de/news/preis-beim-moebelkauf-weniger-wichtig/. Zugegriffen am 27.05.2019.

Verband Schweizer Möbelhandel und -industrie. (о. J.). Umsatzentwicklung der grössten Möbelhändler 2009-2015. Verband möbelschweiz. https://www.xn--mbelschweiz-rfb.ch/de/publikationen/kennzahlen-produktion/moe-belhandel.html. Zugegriffen am 17.06.2018.

Vogt, S., \& Werner, M. (2014). Forschen mit Leitfadeninterviews und qualitativer Inhaltsanalyse. Köln: Fachhochschule Köln.

Winters, P. (2016). Customer Strategy: aus Kundensicht denken und handeln (2. Aufl.). Freiburg/ München/Stuttgart: Haufe Gruppe.

Wright, L.T., Newman, A., \& Dennis C. (2006). Enhancing consumer empowerment. European Journal of Marketing, 40(9/10), 925-935.

Zhang, M., Ren, C., Wang, G. A., et al. (2018). The impact of channel integration on consumer responses in omni-channel retailing: The mediating effect of consumer empowerment. Electronic Commerce Research and Applications, 28, 181-193. https://doi.org/10.1016/j.elerap.2018.02.002.

Zorraquino.com. (o. J.). ROPO (Research Online Purchase Offline). Zorraquino. https://www.zorraquino.com/dictionary/what-isropo-rese-arch-online-purchase-offline.html. Zugegriffen am 05.06.2019. 


\section{Aemmer, David (M.Sc. BA/david_aemmer@gmx.ch).}

Konsekutives Masterstudium in Betriebsökonomie (Vertiefung in Corporate/Business Development) an der Berner Fachhochschule. Langjährige Erfahrung im Qualitäts-, Prozess- und Projektmanagement. Aktuell bei PostNetz tätig als Leiter Projekte Entwicklung Netzformate. Unter anderem zuständig für die Koordination der Projekte rund um die Weiterentwicklung des Kundendienstes der Post. Zusätzlich Leiter des Großprojekts „Filiale der Zukunft“ und dabei verantwortlich für die Entwicklung sowie den Roll-out des neuen Konzepts für die eigenbetriebenen Filialen der Post an über 300 Standorten.

\section{Bigler, Jonas (M.Sc. BA/jonas.bigler@gmx.ch).}

Konsekutives Masterstudium in Betriebsökonomie (Vertiefung in Corporate/Business Development) an der Berner Fachhochschule mit Stipendium der Hirschmann Stiftung. Langjährige Erfahrung als Marketing- und Produktmanager von digitalen und physischen Finanzdienstleistungen im B2B- und B2C-Bereich. Leidenschaftlich interessiert am Internet mit eigenen Projekten bezüglich eSports und Onlinekleinanzeigen (Co-Initiator von nimms.ch, der Plattform für Kostenloses). Aktuell tätig als Senior Product Manager Digital Channels bei der PostFinance AG.

\section{Deane Harder (Prof. Dr./deane.harder@bfh.ch).}

Professor an der Berner Fachhochschule, am Institute for Innovation, Strategy and Entrepreneurship. Schwerpunkte sind Innovation, Verhaltensökonomie und Systemik. Vorher 10 Jahre in der freien Wirtschaft, u. a. in verschiedenen Beratungsunternehmen in den Bereichen Strategie, Innovation, Nachhaltigkeit und Prozessoptimierung, und mehrere Jahre selbstständig als Consultant.

Open Access Dieses Kapitel wird unter der Creative Commons Namensnennung 4.0 International Lizenz (http://creativecommons.org/licenses/by/4.0/deed.de) veröffentlicht, welche die Nutzung, Vervielfältigung, Bearbeitung, Verbreitung und Wiedergabe in jeglichem Medium und Format erlaubt, sofern Sie den/die ursprünglichen Autor(en) und die Quelle ordnungsgemäß nennen, einen Link zur Creative Commons Lizenz beifügen und angeben, ob Änderungen vorgenommen wurden.

Die in diesem Kapitel enthaltenen Bilder und sonstiges Drittmaterial unterliegen ebenfalls der genannten Creative Commons Lizenz, sofern sich aus der Abbildungslegende nichts anderes ergibt. Sofern das betreffende Material nicht unter der genannten Creative Commons Lizenz steht und die betreffende Handlung nicht nach gesetzlichen Vorschriften erlaubt ist, ist für die oben aufgeführten Weiterverwendungen des Materials die Einwilligung des jeweiligen Rechteinhabers einzuholen.

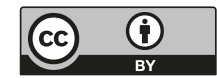

